\title{
Modélisation de la croissance des peuplements réguliers de hêtre : dynamique des hiérarchies sociales et facteurs de production *
}

\author{
JF Dhôte \\ ENGREF, laboratoire de recherches en sciences forestières, \\ 14 rue Girardet, 54042 Nancy, France
}

(Reçu le 10 janvier 1991; accepté le 23 mai 1991)

\begin{abstract}
Résumé - Afin de permettre la simulation de la croissance de peuplements réguliers de hêtre soumis à divers traitements sylvicoles, un modèle indépendant des distances a été mis au point, à partir des données recueillies dans des essais comparatifs d'éclaircie observés sur de longues périodes. On a choisi de modéliser simultanément et de façon cohérente l'effet de la densité et des facteurs sociaux sur la croissance, aux deux niveaux de l'arbre et du peuplement. Le modèle repose sur une double représentation de la compétition interindividuelle. 1) Compétition verticale unilatérale entre classes sociales : le peuplement est subdivisé en deux sous-unités, l'étage principal de végétation et le sous-étage; l'étage principal inhibe totalement la croissance du sous-étage, lequel ne concurrence pas l'étage principal. 2) Compétition horizontale dans l'étage principal : la croissance d'un individu dépend de sa position sociale et de la densité partielle de l'étage principal. L'articulation entre les deux étages a lieu au voisinage d'une dimension-seuil, dont la valeur dépend de l'áge et de la densité du peuplement. Le choix d'une formulation mathématique simple permet l'étude explicite du comportement asymptotique des trajectoires individuelles : le modèle prédit une structuration continue des peuplements, des individus initialement dans l'étage dominant étant progressivement recouverts et relégués dans le sous-étage; ces régressions sociales se manifestent par l'apparition de distributions bimodales. Les prédictions sont testées favorablement pour des situations s'écartant fortement de celles qui ont servi à la construction du modèle. Toutefois, certains écarts constatés entre prédictions et observations laissent penser que la compétition pour la lumière ne suffit pas à rendre compte de la dynamique des différentes classes sociales : des différences de fonctionnement entre périodes de bonne alimentation en eau et périodes de stress hydrique suggèrent l'intervention d'une compétition souterraine. Ces résultats contribueront à la généralisation du modèle pour une large gamme de conditions écologiques et, éventuellement, pour d'autres espèces.'
\end{abstract}

dynamique des peuplements forestiers / hiérarchie sociale / facteur de production / modélisation / niveaux d'organisation / Fagus silvatioa L

Summary - Modelling the growth of even-aged beech stands: dynamics of hierarchical systems and yield factors. In order to buiid a growth simulator for even-aged stands of common beech (Fagus silvatica $L$ ) under various management regimes, a distance-independant individual-tree model was developed, on the basis of repeated measures in long-term thinning trials. The method aimed at representing, in a simultaneous and coherent way, the effect of density and social status on

\footnotetext{
* Cet article reprend et complète les résultats acquis dans le cadre d'un travail de thèse effectué à I'INRA, Station de Sylviculture et de Production, Centre de Recherches Forestières de NancyChampenoux, sous la direction de J Bouchon, N Le Goff et JM Ottorini.
} 
growth, at both tree- and stand-level. The model is based on two assumptions concerning inter-tree competition. 1) One-sided vertical competition between social classes: the stand is organized in two subsystems, the main vegetation storey and the understorey; the overstorey inhibits completely understorey growth, whereas understorey does not influence overstorey. 2) Horizontal competition in the overstorey: the growth of one tree depends on its social position and on the overstorey partial density. The two storeys are separated by a threshold dimension, the value of which depends on age and density of the stand. The rather simple mathematical formulation that was chosen allows an analytical study of the asymptotic behaviour of individual tree trajectories: the model predicts a continuous structuration of stands, as trees initially in the overstorey are progressively overtopped and drop back to the understorey; the consequence of such social regressions is the development of bimodal distributions. The predictions are favourably tested against actual observations, especially in the case of situations differing markedly from those used to build the model. But some discrepancies still subsist between predictions and observations and let us argue that competition for light is not sufficient to account for the differences in the dynamics of various social classes: dissimilar stand functioning between periods of favourable water conditions and periods of water stress indicate that competition also exists between root systems. These results will lead to a more general formulation of the model, for a large range of ecological conditions and, possibly, for other species.

forest stands dynamics / social hierarchies / yield factors / modelling / organisation levels / Fagus silvatica $L$

\section{INTRODUCTION}

La sylviculture des hêtraies connaît actuellement une évolution, principalement due à une meilleure connaissance des facteurs qui déterminent la qualité des produits (Polge, 1973 et 1981; Keller et al, 1976; Ferrand, 1982). Des traitements plus sélectifs et beaucoup plus intensifs que par le passé sont préconisés : dépressages vigoureux opérant une première sélection (Schütz, 1981), désignation précoce d'arbres "d'avenir» et éclaircies fortes à leur profit. Ces pratiques sont susceptibles, en outre, d'accélérer le cycle de production de la hêtraie, en concentrant l'accroissement sur un petit nombre d'individus (Bryndum, 1987). En parallèle à l'analyse d'essais récents, où ces sylvicultures sont expérimentées en vraie grandeur (Bouchon et al, 1989), il est apparu nécessaire de pouvoir disposer d'un modèle de croissance permettant de simuler la réponse des arbres et des peuplements à divers scénarios sylvicoles, sur de longues périodes. De plus, la conduite et la production des futaies régulières de hêtre ont fait l'objet, depuis plus d'un siècle, de l'installation d'un réseau d'expériences locales (Oswald et Divoux, 1978; Pardé, 1981) et le modèle devait contribuer à une synthèse des résultats fournis par ces "placettes permanentes de production». Nous nous proposons de présenter dans cet article un certain nombre de résultats acquis au cours du travail de modélisation, en insistant sur les aspects méthodologiques, biologiques (hypothèses de construction du modèle) et mathématiques; nous reviendrons ultérieurement sur les possibilités d'application pratique du modèle.

On s'intéresse ici aux peuplements réguliers de hêtre, si l'on entend par là des peuplements issus de régénération naturelle ou artificielle, donc sensiblement équiennes, et où le hêtre représente au minimum $80 \%$ du nombre des tiges, c'est-àdire pratiquement monospécifiques. Bien que les données à partir desquelles le modèle a été construit et validé proviennent de 5 grands massifs forestiers répartis 
dans le Nord de la France, la «populationcible" concerne a priori l'ensemble des hêtraies; il faut remarquer d'emblée que le matériel expérimental ne recouvre pas le double gradient climatique et stationnel des hêtraies. Cette situation justifie l'intérêt porté à la définition d'un cadre théorique, qui assure un lien entre la structure du modèle et les facteurs de la production biologique (lumière, eau...). Cependant, la question de l'interaction milieu-sylviculture reste ouverte et impose de poursuivre la validation déjà engagée du modèle : on indiquera quelques perspectives à ce sujet.

D'un point de vue temporel, l'intervalle étudié va du stade du bas perchis à la futaie adulte (30 à 150 ans), phase bien décrite par les placettes permanentes de production. À l'intérieur de cet intervalle, deux échelles de temps seront considérées : au niveau d'un arbre, la trajectoire observée doit être envisagée à court terme, c'est-àdire comme une succession d'accroissements instantanés, dont chacun dépend de conditions locales en évolution permanente et perturbées éventuellement par le traitement sylvicole (l'âge, la densité et la structure du peuplement, la position sociale); en ce sens, un modèle de la croissance individuelle est nécessairement différentiel. Mais, dans le même temps, la connaissance des comportements à long terme conduit à dégager des structures temporelles qui seraient difficiles à détecter avec les seuls accroissements instantanés : en cela réside tout l'intérêt des dispositif́s suivis sur des longues périodes. Enfin, c'est sur toute la durée d'une révolu: tion qu'on veut évaluer et comparer la réponse des peuplements aux traitements : le modèle (différentiel) doit être fiable dans le cadre de prévisions à longue échéance, ce qui explique qu'on ait accordé une importance particulière à des aspects mathématiques : simplicité de la formulation du modèle, étude des comportements asymptotiques.
En ce qui concerne les sylvicultures, le modèle doit être assez souple pour permettre la simulation de divers scénarios : séries d'éclaircies variables par la périodicité, l'intensité et la nature des coupes. Pour satisfaire cette exigence, on a opté pour une approche du type modèle d'arbre indépendant des distances (Munro, 1974; Houllier, 1986; Houllier et al, 1991), qui consiste à représenter la croissance de chaque arbre, compte tenu de son rang social et de l'âge, de la densité et de la structure du peuplement. Avec ce type de modèle, on peut notamment prédire l'évolution de la distribution des dimensions individuelles, et donc simuler fidèlement l'impact d'éclaircies plus ou moins intenses et de différentes natures (par le bas, par le haut, etc). De plus, la localisation des arbres dans l'espace n'est pas requise pour rentrer dans le modèle, ce qui est compatible avec la nature des données disponibles dans les placettes permanentes. Les deux principales limitations du matériel expérimental utilisé sont d'une part la rareté des données individuelles avant l'âge de 60 ans (phase très importante d'un point de vue sylvicole), d'autre part la faible amplitude des traitements pratiqués (les densités ne sont jamais très faibles, dans les placettes permanentes) : en ce sens, les utilisations prévues du modèle seront, dans une certaine mesure, exploratoires (les traitements qu'on veut simuler se situeront en marge du domaine expérimenté, voire à l'extérieur), ce qui renforce la nécessité d'hypothèses biologiques fortes et explicites dans la formulation du modèle.

\section{MATÉRIEL ET MÉTHODES}

Les données sur lesquelles s'appuie cette étude proviennent du réseau français de placettes permanentes de production pour le hêtre (Oswald, 1971 et 1981; Oswald et Divoux, 1978; Pardé, 1981). Ce réseau comprend notamment des es- 
sais d'éclaircies, groupes de placettes organisées en dispositifs, avec plusieurs modalités testées dans un bloc homogène quant au milieu et au peuplement initial. Les dispositifs sont installés dans 5 grandes forêts domaniales : Eawy (Seine-Maritime), Retz (Aisne), Souilly (Meuse), Haye (Meurthe-et-Moselle) et Darney (Vosges).

A l'origine, les peuplements étaient tous issus de régénération naturelle, très denses (à l'exception de Souilly et Eawy), et le hêtre y représentait entre 80 et $90 \%$ du nombre de tiges total.

\section{Traitements pratiqués}

Haye : 5 placettes installées en 1883, à l'âge de 28 ans. II y a en fait deux dispositifs distants de quelques centaines de mètres, mais manifestement sur stations et de productivités identiques : places dites de la route Charlemagne ( 3 placettes de 25 ares) et de la route de Chavigny (2 placettes de 20 ares), qui seront regroupées ici. Les traitements consistent en 1 témoin (on n'exploite que les arbres morts ou dépérissants), 2 placettes éclaircies par le bas et 2 placettes éclaircies par le haut. Les coupes ont été très légères jusqu'à 60 ans (forte mortalité en sous-étage dans le témoin et les éclaircies par le haut), ensuite les éclaircies par le haut conduisent à des peuplements plus clairs (fig 1). Pour tous les traitements, les densités restent fortes, au niveau d'éclaircies faibles à modérées si l'on compare avec la table de production allemande de Schober (1972), classe de productivité 9 . Tous les individus survivants ont été numérotés en 1919 (64 ans); auparavant, on dispose d'inventaires par classes de circonférence.

Retz : le dispositif du Faîte, qui compte 4 placettes de 20 ares, a été installé en 1925 (âge 35 ans) et constitue un essai de traitements à rotations différentes : les rotations de $3,6,9$ et 12 ans ont été appliquées assez régulièrement, sauf les plus courtes pour lesquelles les éclaircies s'espacent à partir de 60 ans. Les densités des 4 placettes divergent jusqu'à 50 ans puis se rejoignent vers 80 ans (même avec des éclaircies modérées, les rotations de 3 et 6 ans constituent un traitement relativement intensif, alors que les rotations longues conduisent à des peu- plements denses). Dans tous les cas, les éclaircies sont plutôt par le haut. Tous les arbres de circonférence supérieure à $20 \mathrm{~cm}$ ont été numérotés dès l'installation. Ces données individuelles sont complétées par des inventaires concernant toutes les tiges.

Eawy : le dispositif de Camp Cusson compte 4 placettes de 1 ha; installation en 1924, à 44 ans. Les éclaircies sont par le haut et diffèrent à la fois par la rotation et l'intensité : 1 , éclaircies modérées tous les 5 ans ("à la danoisen); 2, éclaircies faibles tous les 10 ans; 3, éclaircies fortes tous les 10 ans; 4 , éclaircies «suivant la méthode locale" (proches de 2). Un écart de densité a été bien maintenu, dès l'origine, entre les placettes 1 et 3 d'une part, 2 et 4 d'autre part. Tous les arbres ont été numérotés en 1934 (54 ans).

Seuls ces trois groupes de placettes ont été considérés lors de la construction du modèle, en raison de l'existence de séries chronologiques de mesures individuelles. Les deux expériences de Souilly et de Darney (Sainte Marie) (voir $\mathrm{Li}$ mites de linterprétation par la compétition aérienne), où l'on ne dispose que des séries d'inventaires par classes de diamètre ou de circonférence, ont été réservées pour la phase de validation.

\section{Mesures et notations}

Les mesures ont été effectuées seulement, à quelques exceptions près, aux dates de passage en éclaircie : on dispose donc de 5 à 11 mesures par placette, suivant le traitement appliqué. Sur chaque individu est relevée la circonférence à $1,30 \mathrm{~m}$; la hauteur et le volume sont mesurés sur des échantillons dont la qualité n'est pas toujours suffisante pour qu'on puisse faire des estimations fiables pour l'ensemble du peuplement. Tout le travail a donc porté sur les grandeurs à 1,30 m (circonférence, surface terrière). Des notations de statut social ont été effectuées à deux reprises, mais se sont avérées peu exploitables.

Notations : on utilisera les majuscules pour désigner les statistiques de peuplement usuelles (nombre de tiges $N$, surface terrière $G$ ) et les minuscules pour les grandeurs individuelles : pour un arbre, $c$ sera la circonférence à $1,30 \mathrm{~m}$ et $\mathrm{g}$ la surface terrière correspondante. 

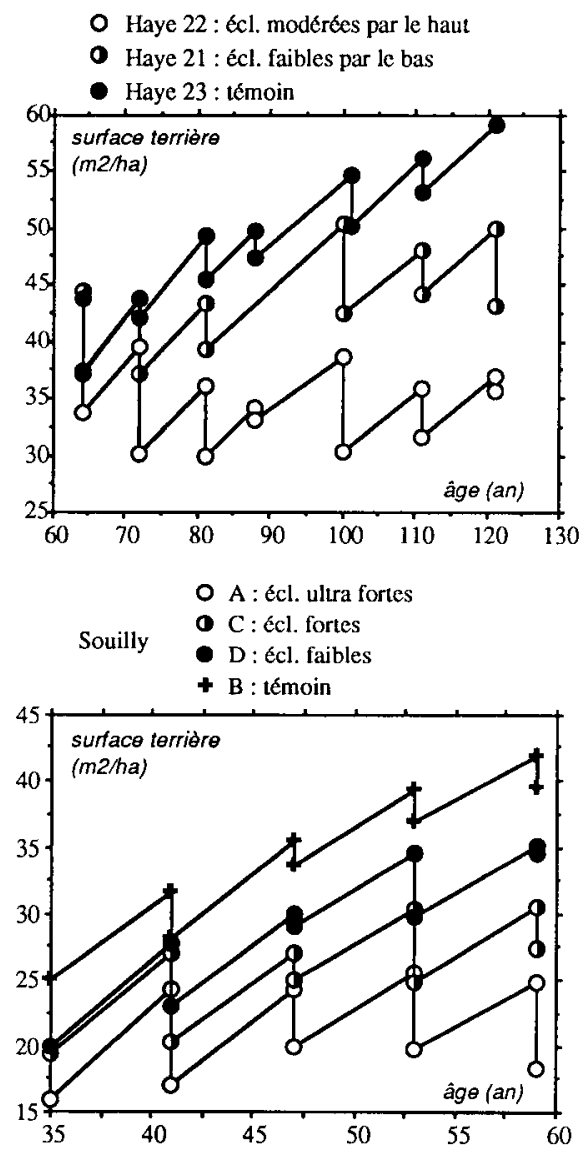

\section{DE L'ANALYSE DES DONNÉES À LA FORMULATION D'HYPOTHĖSES}

\section{Conservation de la production aux fortes densités}

Le réseau expérimental présente systématiquement des groupes de placettes qui, temporairement ou de façon durable, sont maintenues à des densités différentes (fig 1). En définissant la production totale en surface terrière d'un peuplement comme la somme de ses accroissement
O Eawy 3 : écl. fortes tous les 10 ans

- Eawy 1 : écl. modérées tous les 5 ans

- Eawy 2 : écl. faibles tous les 10 ans

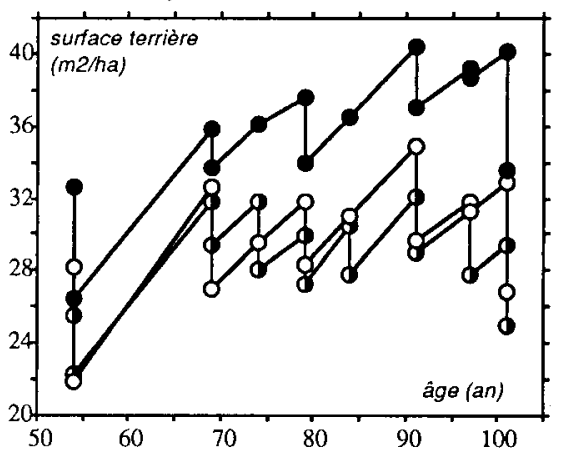

Fig 1. Surface terrière sur pied, en fonction de l'áge, pour 3 dispositifs en forêts de Haye, Eawy et Souilly. Les discontinuités figurent les éclaircies; la mortalité naturelle pendant chaque période est comptée dans l'éclaircie qui suit.

depuis l'origine, y compris la mortalité naturelle, on constate que, sur de très longues périodes, la production totale à l'intérieur d'un dispositif donné est quasiment identique pour tous les traitements, et notamment indépendante de la densité (tableau I). La nature des éclaircies (par le haut ou par le bas) n'a pas d'influence détectable, non plus que le caractère précoce ou tardif des interventions. Dans le dispositif en carré latin de Souilly, qui comprend 4 modalités de traitement bien différenciées en densité, répétées 4 fois, il n'y a pas d'effet significatif du traitement sur 
l'accroissement brut en surface terrière (mortalité comprise) entre 35 et 59 ans.

Ces résultats sont à replacer dans le contexte des nombreuses études menées par ailleurs sur l'effet de la densité sur la croissance globale (du peuplement) : on peut résumer la littérature consacrée à ce sujet par le modèle très schématique de la figure 2 (d'après Johnston et al, 1967). On appelle densité maximale biologique $\delta_{\max }$, celle d'un peuplement plein, en autoéclaircie. Lorsque la densité varie dans certaines limites (intervalle $\left[\delta_{c r}, \delta_{\max }\right]$ ), la croissance brute du peuplement (ie mortalité incluse) varie peu, ce que schématise le plateau de la figure 2 : ce cas correspond typiquement à la situation des essais d'éclaircies dans des peuplements initiale-

Tableau I. Surface terrière sur pied et accroissement annuel en surface terrière, pour les traitements des 3 dispositifs de Haye, Eawy et Souilly (traitements définis dans Matériel et méthodes). Les valeurs mentionnées sont des moyennes sur la période de référence indiquée.

\begin{tabular}{llll}
\hline $\begin{array}{c}\text { Période } \\
\text { de } \\
\text { référence }\end{array}$ & Placette & $\begin{array}{c}\text { Surface } \\
\text { terrière } \\
\text { sur pied } \\
\left(e n m^{2}\right. \\
\left.h a^{-1}\right)\end{array}$ & $\begin{array}{c}\text { Accroissement } \\
\text { annuel en } \\
\text { surface terrière } \\
\left(e^{-n} m^{2}\right. \\
\left.h a^{-1} a^{-1}\right)\end{array}$ \\
\hline \multicolumn{5}{c}{ De 64 } & Haye 23 & 49,0 & 0,645 \\
à 121 ans 21 & 43,6 & 0,613 \\
& Haye 11 & 43,8 & 0,572 \\
& Haye 12 & 38,9 & 0,653 \\
De 54 & Haye 22 & 34,2 & 0,569 \\
à 101 ans & Eawy 2 & 36,3 & 0,494 \\
& Eawy 3 & 30,2 & 0,554 \\
& Eawy 1 & 29,2 & 0,536 \\
De 35 & Souilly B & 34,0 & 1,02 \\
à 59 ans & Souilly D & 28,7 & 1,06 \\
& Souilly C & 25,6 & 1,05 \\
& Souilly A & 21,5 & 1,10 \\
\hline
\end{tabular}

ment pleins (Møller, 1954; Pardé, 1964 1981). Lorsque la densité est inférieure à une valeur critique $\delta_{c r}$, la croissance varie cette fois proportionnellement à la densité; on retrouve ici un autre cas typique qui est celui des essais d'espacement dans les plantations (Braathe, 1957). Nous parlerons, pour désigner cette réponse du peuplement à la densité, de limitation et de saturation, les faibles densités étant limitantes pour la croissance, alors que les fortes sont saturantes. Au niveau de l'arbre, la situation est inverse : la croissance individuelle est maximale et constante en dessous de $\delta_{c r}$, alors qu'elle diminue régulièrement au-delà.

Un article récent de Whyte et Woolons (1990) montre bien comment, à partir d'un large gradient initial de densités dans des plantations de Pinus radiata, on passe progressivement au cours du temps d'une situation de densité limitante à une situation de saturation, et ce d'autant plus lentement que la densité initiale est plus faible. Un autre exemple de gradient de densité savamment maintenu et de son effet sur la croissance est fourni, pour le hêtre, par l'expérience de Totterup (Bregentved, Danemark : Bryndum, 1987), dont nous nous inspirerons pour la validation du modèle (cf Qualité de la réponse prédite à la densité).

Une abondante controverse a couru depuis un demi-siècle parmi les forestiers européens sur la forme exacte de la relation densité-croissance du peuplement : s'agitil d'une réponse monotone croissante ou à optimum (Langsæter, 1941, Assmann, 1970); y a-t-il une densité critique (fig 2) ou bien passe-t-on progressivement d'un régime à l'autre; $y$ a-t-il des différences entre espèces, entre éclaircies par le bas et par le haut, entre la croissance en volume et en surface terrière (synthèse par Braathe 1957, expérience récente sur le Douglas (Pseudotsuga menziesii) par Curtis et Marshall, 1986). 


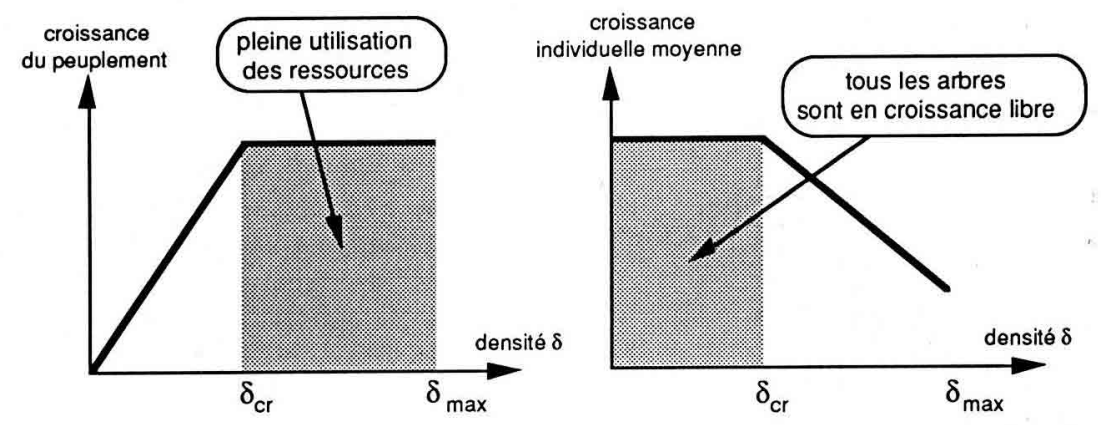

Fig 2. Représentation schématique de l'effet de la densité sur la croissance, aux deux niveaux de l'arbre et du peuplement (d'après Johnston et al, 1967).

Les deux schémas, arbre et peuplement, sont généralement interprétés en invoquant un mécanisme de compétition pour l'accès aux ressources du milieu, principalement l'occupation de l'espace aérien (Braathe, 1957, Johnston et al, 1967; Long, 1985) : on dira qu'aux faibles densités, il n'y a pas d'interactions compétitives entre les individus (croissance libre), qui utilisent tout l'espace disponible; dès que le couvert se referme, c'est le peuplement globalement qui fait un usage maximal de l'espace et la croissance individuelle est limitée par la compétition aérienne.

Les données observées de production en surface terrière, dans les placettes permanentes de hêtre, supportent donc l'hypothèse d'une réponse à la densité de type saturation. De plus, l'intervalle minimal dans lequel la production est quasiinvariante, entre $1 / 2$ et 1 fois la densité maximale biologique, est cohérent avec les valeurs mentionnées par Møller (1954) pour la même espèce. Enfin, si l'on tient à ces seules données au niveau peuplement, les situations testées sont toutes saturantes et ne permettent pas d'estimer l'ensemble de la réponse, notamment dans l'intervalle des densités limitantes.

\section{Croissance individuelle et rang social : effet de seuil}

À partir des séries chronologiques de mesures relevées pour chaque arbre, on a examiné les vitesses de croissance individuelles en surface terrière, pour chaque placette et chaque période entre 2 mesures (c'est-à-dire en tout 74 périodes pour les 13 placettes). Quand on observe cette vitesse en fonction de la circonférence initiale au début de la période considérée, une structure très nette se dégage, avec deux intervalles distincts (fig 3) : aux faibles dimensions, relativement au reste du peuplement, la vitesse est nulle en moyenne et la variabilité est faible; au-delà d'une certaine dimension, la vitesse est significativement positive, varie linéairement avec la circonférence et la variabilité résiduelle est forte.

L'articulation entre les deux intervalles n'est pas progressive, mais s'apparente en 
accroissement annuel en sufface terrière ( $\mathrm{cm} 2 / \mathrm{an})$

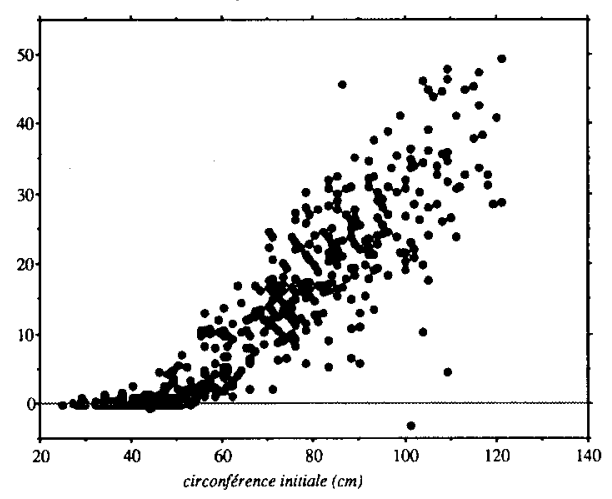

Fig 3. Vitesse de croissance individuelle en surface terrière, entre 54 et 69 ans, en fonction de la circonférence initiale (à 54 ans); sont représentés les 538 arbres de la placette Eawy3 (surface 1 ha).

général à une transition brutale, ce qui nous a conduit à introduire la notion de seuil : dans un peuplement donné, il existe un valeur $\sigma$ de la circonférence telle que la croissance d'un individu quelconque obéit au modèle structurel suivant :

$$
\frac{\mathrm{d} g}{\mathrm{~d} t}=\gamma(c-\sigma) \text { si } c \geq \sigma, \quad 0 \text { sinon }
$$

où $c$ et $g$ sont la circonférence et la surface terrière de l'individu considéré et $\gamma$ un paramètre (pente de la droite).

Cette structure des accroissements est remarquablement stable, quels que soient l'âge et la densité des peuplements. Pour le montrer, nous présentons à la figure 4 des superpositions de représentations analogues à la figure 3 , à la différence que les données individuelles sont regroupées en représentant des moyennes par classe (pour chaque placette et chaque date de mesure, nous avons regroupé les $N$ individus présents, ordonnées par circonfé- rence, en $K$ classes de même effectif $N / K$, avec $K=10$ à la figure 4).

La densité influe peu sur la valeur du seuil $\sigma$ : par exemple, on peut estimer qu'elle prend la même valeur pour les deux placettes Eawy2 (dense) et Eawy3 (claire) (fig 4A). La croissance des arbres dominés et surcimés est très faible et peu influencée par le traitement; au contraire, dans l'étage de végétation principal (dominants et codominants), la stimulation de la croissance à faible densité est nette, quel que soit le statut social; mathématiquement, tout se passe comme si, dans le modèle structurel (eqn 1), la densité ne jouait pas sur le seuil $\sigma$, mais uniquement sur la pente $\gamma$.

Lorsqu'on compare des couples éclaircies par le bas-par le haut (données non figurées ici), la même structure qu'à la figure 4A apparaît, les éclaircies par le haut conduisant généralement à des peuplements plus clairs (Pardé, 1981). La nature des éclaircies n'a pas, en soi, d'effet sur la valeur du seuil $\sigma$ (il faut noter que $\sigma$ correspond, pour des éclaircies par le bas, à une dimension en quelque sorte «inusitée»: tous les arbres de dimension voisine de ou inférieure à $\sigma$ ont disparu en éclaircie...).

Avec l'âge, par contre, la valeur de $\sigma$ augmente régulièrement (fig 4B), et l'on a constaté, empiriquement, que $\sigma$ varie sensiblement comme la moitié de la circonférience dominante $c_{0}$. Ainsi, on vérifie que le rapport de dominance proposé par Alder (1979), qui pour un individu de circonférence $c$, s'exprime $c / c_{0}$, est une quantité qui détermine assez bien son statut social, indépendamment de l'âge et de la densité.

\section{Interprétation et hypothèses}

Au niveau du peuplement, la croissance brute en surface terrière est pratiquement 


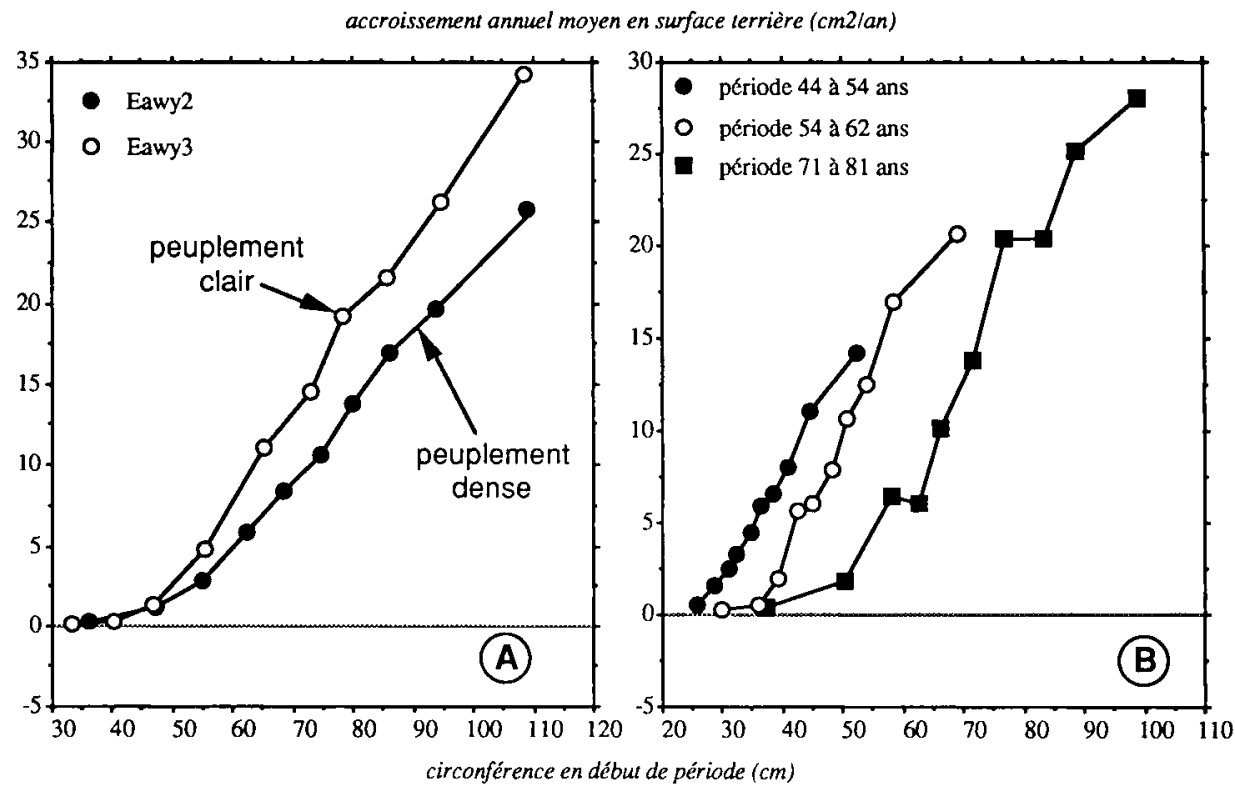

Fig 4. Vitesse de croissance individuelle et dimension initiale : effet de la densité et de l'âge en $A$ : comparaison de deux placettes de densités différentes, sur une même période de croissance (54-69 ans); en B : comparaison de trois périodes de croissance successives, pour une même placette (Retz3). Les individus sont regroupés en 10 classes de même effectif, pour chaque placette et chaque période considérée, et les données figurées sont les moyennes par classe.

indépendante de la densité, dans certaines limites, tandis qu'au niveau de l'arbre, un effet rang social basé sur la notion de seuil a été mis en évidence. On peut justifier ces deux constats, ainsi que les lois de comportement du seuil avec la densité et l'âge, en ayant recours à la description de la structure verticale du couvert et au phénomène d'interception de la lumière. Dans un peuplement régulier fermé, l'énergie lumineuse incidente s'atténue très rapidement entre le sommet et la base du couvert, en raison de l'interception par les parties supérieures du feuillage. Le profil vertical de l'énergie est généralement bien relié à la distribution de la masse foliaire (loi de Beer-Lambert : Landsberg, 1986). Pour donner un exemple, l'étude menée par Aussenac et al (1982), sur des jeunes plantations de Douglas, a montré que, même dans le cas d'éclaircies intenses (1 rang sur 2), l'interception totale d'énergie, au niveau de la base de l'étage principal de végétation, est presque complète.

Dès lors, si la production de biomasse par le peuplement n'est limitée que par l'interception lumineuse, on conçoit qu'elle puisse être identique pour des peuplements ayant des masses foliaires (et a fortiori des densités) allant du simple au double : c'est assez exactement l'intervalle dans lequel on se trouve pour les placettes 
de hêtre (encore convient-il de préciser qu'il est rare qu'une seule éclaircie ait divisé la densité par deux...). Ces considérations qualitatives sont très approximatives, dans la mesure où elles négligent les différents termes du bilan de carbone dont résulte la production nette de biomasse (photosynthèse brute, respirations, recyclage de matière), mais on doit préciser qu'elles sont cohérentes avec les simulations de modèles théoriques de production et d'allocation de la biomasse entre organes (voir, notamment, McMurtrie, 1985).

Le résultat mentionné est également intéressant au niveau individuel : un arbre dominé ou surcimé, dont tout le houppier est au mieux à la base du couvert principal, voire en dessous, ne bénéficie que d'un éclairement réduit et diffus et sa situation n'est pas significativement améliorée, même par une éclaircie forte. Au contraire, les individus suffisamment dominants, qui ont accès à la lumière incidente directe, ont un potentiel d'assimilation qui leur permet de maintenir leur position sociale et de poursuivre une croissance soutenue (Ford, 1975).

L'interprétation par l'interception de la lumière permet donc d'abord de justifier l'articulation brutale (effet de seuil) entre les deux étages de végétation superposés : ce que nous appelons "seuil» correspondrait à la taille minimale des individus de l'étage dominant; on comprend dès lors que cette quantité augmente avec l'âge, c'est-à-dire avec le développement général du peuplement. En outre, cette interprétation suggère implicitement un aspect dynamique des hiérarchies sociales : le recouvrement d'un arbre, initialement dans l'étage dominant, par ses voisins plus vigoureux est un phénomène rapide, suivi éventuellement (pour une espèce d'ombre comme le hêtre) par une longue phase de stagnation dans le sous-étage (Ford et
Newbould, 1970); ce phénomène d'oppression continue se traduit par le fait que la distribution des dimensions individuelles devient bimodale, phénomène dont les placettes permanentes de hêtre offrent plusieurs exemples très nets.

\section{Généralisation}

À partir de ces hypothèses, il est possible d'imposer aux composantes du modèle des lois de comportement plus générales que celles suggérées par le jeu de données étroit que nous utilisons ici. Dans le cas des faibles densités, le couvert est très fortement ouvert et l'énergie lumineuse n'est plus totalement interceptée par le peuplement, ce qui justifie une baisse de la croissance globale. Au niveau de l'arbre, l'intensité des interactions entre classes sociales diminue brutalement, dès que la densité est suffisamment faible : il n'y a plus de couvert continu, la stratification verticale des individus perd de son importance puisque tous, quelle que soit leur dimension, bénéficient de conditions d'éclairement satisfaisantes; mathématiquement, nous traduisons ce comportement en imposant au seuil de tendre vers 0 lorsque la densité tend vers 0 .

Sur ce dernier point (possibilité de réaction à l'éclaircie d'arbres dominés, sans délai), il faut remarquer que le raisonnement suppose implicitement une grande plasticité sylvicole. Ainsi, un arbre longtemps concurrencé et brutalement mis en lumière peut présenter un syndrome d'adaptation (déséquilibre hydrique lié à une transpiration accrue, déséquilibre mécanique lié à la disparition de l'entourage). Dans le cas du hêtre, cette plasticité a été confirmée dans des essais récents (Bouchon et al, 1989 et données non publiées) pour des arbres dominants et codominants; de plus, des reprises d'accroisse- 
ment très nettes ont été observées chez des individus initialement surcimés, dans le cas d'une forte mise en lumière. Mais il reste probable que ces phénomènes d'adaptation et de délais de réaction soient en partie responsables des différences de réaction à l'éclaircie, fréquemment mentionnées entre espèces de tempéraments différents.

\section{Prise en compte du milieu}

La quasi-invariance constatée de la production totale en surface terrière en fonction du traitement permet de supposer que cette quantité ne dépend que de la station. Chaque site étudié sera donc caractérisé par une production totale maximale $\Gamma$, qui est une fonction de l'âge. De plus, on supposera que le milieu n'a pas d'influence sur les paramètres du modèle au niveau arbre (paramètres $\lambda$ et $\mu$ dans Compatibilité entre les deux niveaux), qui sont donc des paramètres globaux. Ainsi, les seuls paramètres locaux seront ceux intervant dans $\Gamma$.

Cette paramétrisation signifie que le milieu n'influe que sur le profil général de la croissance globale, au cours du temps, mais pas sur la réponse individuelle au traitement. En d'autres termes, il n'y a pas d'effet d'interaction milieu-sylviculture. Cette hypothèse très simple paraît cohérente avec le jeu de données pris en compte pour la construction du modèle qui concerne, répétons-le, de très bonnes stations dans les 3 massifs forestiers de Haye (Lorraine), Retz (Picardie) et Eawy (Normandie). Elle constitue également l'hypothèse la plus prudente tant qu'on ne disposera pas simultanément d'un modèle assez fiable et de données assez larges pour tester d'éventuelles différences de comportement entre milieux, au niveau de l'arbre.

\section{CONSTRUCTION DU MODĖLE ET COMPORTEMENT MATHÉMATIQUE}

\section{Compatibilité entre les deux niveaux}

Au niveau du peuplement, la vitesse de croissance en surface terrière dépend de l'âge, du milieu et de la densité selon le modèle suivant :

$$
\frac{\mathrm{d} G}{\mathrm{~d} t}=\frac{\mathrm{d} \Gamma}{\mathrm{d} t}\left(1-\mathrm{e}^{-v \mathrm{dens}}\right)
$$

où dens est une mesure de densité à déterminer (voir plus loin dans ce $\$$ ) et $v$ un paramètre; dans cette expression, $\mathrm{d} \Gamma / \mathrm{d} t$ représente la croissance maximale obtenue pour une densité (théorique) infinie.

La croissance en surface terrière d'un arbre quelconque de circonférence $c$ s'écrit, en modifiant le modèle (eqn 1)

$$
\frac{\mathrm{d} g}{\mathrm{~d} t}=\frac{\mathrm{d} \Gamma}{\mathrm{d} t} \alpha(\text { dens })(c-\sigma) \text { si } c \geq \sigma, 0 \text { sinon (2) }
$$

où $\alpha$ est une fonction de la densité.

Soulignons que, pour les deux niveaux, la variabilité de la vitesse de croissance en fonction de l'âge et du milieu intervient de façon multiplicative, à travers le terme $\mathrm{d} \Gamma / \mathrm{d} t$. Dans la croissance d'un arbre, on distingue un effet de la taille (circonférence $c)$, de la dominance, mesurée par l'indice $c / \sigma$, et de la densité à travers la fonction $\alpha$.

Pour spécifier complètement le modèle, nous nous basons sur la nécessité d'assurer la compatibilité entre les niveaux de l'arbre et du peuplement : la somme des accroissements individuels doit être identique au modèle peuplement. On a : 


$$
\begin{aligned}
& \frac{\mathrm{d} G}{\mathrm{~d} t}=\sum_{\text {arbre } i} \frac{\mathrm{d} g_{i}}{\mathrm{~d} t} \quad \text { soit } \frac{\mathrm{d} \Gamma}{\mathrm{d} t}\left(1-\mathrm{e}^{-v \text { dens })}\right. \\
& =\sum_{\text {arbre } i, c_{i} \geq \sigma} \frac{\mathrm{d} \Gamma}{\mathrm{d} t} \alpha \text { (dens) }\left(c_{i}-\sigma\right)
\end{aligned}
$$$$
\text { d'où } 1-e^{-v} \text { dens }=\alpha \text { (dens) }\left(\Sigma_{\sigma}-\sigma N_{\sigma}\right)
$$

où $N_{\sigma}$ est le nombre d'arbres plus gros que le seuil $\sigma$ et $\Sigma_{\sigma}$ la somme de leurs circonférences.

La contrainte de compatibilité entre les deux niveaux (eqn 3) fait apparaître la quantité $\Sigma_{\sigma}$, qui s'est avérée bien expliquer les variations de $\alpha$ et a donc été adoptée comme mesure de densité. Cette mesure est doublement non standard, dans la mesure où il s'agit d'une somme de circonférences, grandeur peu usuelle en dendrométrie (voir, cependant, Maugé, 1975), et où cette somme ne porte que sur une distribution censurée (c'est-à-dire sur les seules valeurs supérieures à un seuil $\sigma$ ). Ce dernier aspect de la mesure permet de revenir sur la façon dont la compétition entre individus est décrite dans le modèle : le peuplement est structuré en deux strates, ou sous-unités fonctionnelles, l'étage principal de végétation et le sousétage; ce dernier n'exerce pas d'influence compétitive sur l'étage principal, lequel au contraire crée des conditions microclimatiques défavorables, qui inhibent totalement la croissance du sous-étage; on dira que la compétition entre les deux strates est "unilatérale» (one-sided competition, Ford et Diggle, 1981). II existe, au sein de l'étage principal, une compétition interindividuelle pour l'accès à la lumière, de nature plus horizontale, dont l'intensité moyenne est décrite par la densité partielle de cet étage, $\Sigma_{\sigma}$.

Nous supposons de plus que la valeur du seuil $\sigma$ peut être déterminée sans ambi- guïté par l'équation implicite :

$$
\sigma=\beta\left(\Sigma_{\sigma}\right) \bar{c}_{\sigma}
$$

où $\bar{c}_{\sigma}$ représente la circonférence moyenne des arbres plus gros que $\sigma$.

La contrainte de compatibilité s'écrit alors :

$$
\begin{aligned}
1-\mathrm{e}^{-v \Sigma_{\sigma}} & =\alpha\left(\Sigma_{\sigma}\right)\left(\Sigma_{\sigma}-\beta\left(\Sigma_{\sigma}\right) \bar{c}_{\sigma} N_{\sigma}\right) \\
& =\alpha\left(\Sigma_{\sigma}\right) \Sigma_{\sigma}\left(1-\beta\left(\Sigma_{\sigma}\right)\right)
\end{aligned}
$$

On a constaté que $\alpha$ pouvait être modélisée par une fonction décroissante légèrement convexe, et l'on a posé :

$$
\begin{aligned}
& \alpha\left(\Sigma_{\sigma}\right)=\lambda e^{-\mu \Sigma_{\sigma}} \\
& \text { et donc } \beta\left(\Sigma_{\sigma}\right)=1-\frac{1-e^{-v \Sigma_{\sigma}}}{\lambda \Sigma_{\sigma} e^{-\mu \Sigma_{\sigma}}}
\end{aligned}
$$

Pour que le seuil $\sigma$ tende vers 0 lorsque la densité tend vers 0 , on peut montrer qu'il est nécessaire d'imposer une relation structurelle entre les paramètres globaux $\lambda, \mu, v$ du modèle $: \lambda=v$.

Cette relation est importante en raison du lien très fort qu'elle établit entre les modèles aux niveaux de l'arbre et du peuplement : non seulement ces deux modèles sont exactement compatibles (par sommation de l'un sur tous les individus, on retrouve l'autre), mais encore le comportement qu'on impose aux différentes composantes du modèle (notamment au seuil $\sigma$ ) induisent un lien entre les paramétrages aux deux niveaux; il n'y a plus de paramètres spécifiques du niveau peuplement, qui est ainsi totalement déterminé par le niveau arbre.

La première conséquence est de réduire le nombre de degrés de liberté : on n'a besoin que de deux paramètres globaux pour spécifier le modèle, ce qui est favorable pour obtenir des estimations 
fiables à partir d'une base expérimentale étroite. La seconde conséquence est de permettre une extrapolation du modèle audelà du domaine pris en compte pour sa construction : répétons que les données disponibles concernent exclusivement des situations de densité saturante, et l'on ne peut donc pas espérer estimer la réponse globale à partir des seules données peuplement; par contre, dans ce même intervalle expérimenté, la réponse individuelle à la densité, telle que mesurée par la fonction $\alpha$ (eqn 5) est tout à fait nette et susceptible d'être modélisée avec une précision acceptable; ainsi c'est grâce au niveau de l'arbre qu'on peut rendre compte de façon satisfaisante, au moins qualitativement, du niveau peuplement.

Nous avons donc retenu la formulation suivante :

$$
\frac{\mathrm{d} g}{\mathrm{~d} t}=\frac{\mathrm{d} \Gamma}{\mathrm{d} t} \alpha\left(\Sigma_{\sigma}\right)\left(c-\beta\left(\Sigma_{\sigma}\right) \bar{c}_{\sigma}\right)
$$

si $c \geq \sigma, \quad 0$ sinon,

$$
\begin{gathered}
\text { où } \quad \alpha\left(\Sigma_{\sigma}\right)=\lambda e^{-\mu \Sigma_{\sigma}} \\
\text { et } \quad \beta\left(\Sigma_{\sigma}\right)=1-\frac{1-e^{-\lambda \Sigma_{\sigma}}}{\lambda \Sigma_{\sigma} e^{-\mu \Sigma_{\sigma}}}
\end{gathered}
$$

et où $\sigma$ est déterminé implicitement par l'équation 4. Unités : $\mathrm{d} g / \mathrm{d} t$ en $\mathrm{m}^{2} \mathrm{an}^{-1}, c, \sigma$ et $\bar{c}_{\sigma}$ en $\mathrm{cm}, \mathrm{d} \Gamma / \mathrm{d} t$ en $\mathrm{m}^{2} \mathrm{ha}^{-1} \mathrm{an}^{-1}, \Sigma_{\sigma}$ en $\mathrm{cm} \mathrm{ha}-1, \lambda$ et $\mu$ en $\mathrm{cm}^{-1}$ ha.

Pour plus de détails concernant la mise en œuvre pratique du modèle et la procédure d'identification, on se reportera à Dhôte (1990, 124-144); précisons seulement que : dès que l'on connaît les paramètres $\lambda$ et $\mu$ ainsi que la distribution des circonférences individuelles, on peut déterminer une valeur $\sigma$ satisfaisant à (4): un algorithme standard de recherche de solu- tion, à partir d'une estimation initiale, permet d'évaluer $\sigma$; dans les applications, le modèle différentiel est intégré par pas de temps fixe; les résultats sont peu sensibles à la longueur du pas, pourvu que celui-ci ne soit pas trop grand (entre 1 et 5 ans, en pratique); en ce qui concerne l'identification du modèle, les deux paramètres globaux $\lambda$ et $\mu$ ont été estimés simultanément sur l'ensemble des données des 3 sites. L'ajustement n'a pas porté sur l'ensemble des données individuelles, mais sur des moyennes par classe (cf Croissance individuelle et rang social : effet de seuil) : avec 10 classes de même effectif par placette et 74 combinaisons placette-période d'accroissement observée (soit 740 «individus"), on obtient les résultats suivants : écart type résiduel sur $\mathrm{dg} / \mathrm{d} t$ : $1,64 \mathrm{~cm}^{2}$ an $^{-1} \quad R^{2}=0,97$

valeur estimée pour $20000 \pi \lambda\left(\mathrm{cm}^{-1} \mathrm{ha}\right)$ : 7,611 ; écart type estimé 0,227

$10000 \mu\left(\mathrm{cm}^{-1} \mathrm{ha}\right): 0,1437$; écart type estimé 0,00728 .

\section{Comportement mathématique : séparation du peuplement en deux sous-populations}

Une question fréquemment invoquée à propos des modèles différentiels est celle de leur fiabilité dans le cadre de prédictions à long terme (ordre de grandeur : plusieurs dizaines d'années). Nous avons choisi d'aborder pragmatiquement ce problème, avec deux points de vue : le premier a consisté à faire une validation systématique du modèle sur de longues périodes, en comparant les simulations à des données observées (voir Validation du modèle sur des données extérieures). Le second point de vue, plus qualitatif, que nous allons développer ici, concerne l'étude générale des trajectoires individuelles, par intégration explicite des équa- 
tions différentielles. Cette étude s'est avérée particulièrement gratifiante dans la mesure où, non seulement on a pu évaluer les propriétés asymptotiques de ces trajectoires, mais on a fait apparaître un aspect du comportement global du système, non prévu à l'origine, qui tient à la dynamique des hiérarchies sociales.

Le modèle opérationnel, sous sa forme (eqn 6), ne se prête pas au calcul explicite, principalement en raison de l'existence de la valeur seuil $\sigma$. En effet, cette valeur, qui joue le rôle d'un paramètre dans l'équation différentielle, est en fait obtenue de façon complexe et implicite, à partir de la distribution des dimensions individuelles et des paramètres du modèle. Pour pouvoir calculer, on a fait quelques simplifications.

Conformément aux observations, les valeurs de $\sigma$ simulées par le modèle varient assez peu, dans un très large intervalle de densités; de plus, ces valeurs évoluent avec l'âge de façon régulière, comme une fraction $1 / 2$ de la circonférence dominante ou encore comme une fraction $\varepsilon$ de la production totale maximale $\Gamma(\varepsilon \approx 0,8)$. Dans un tout autre ordre d'idée, il est aisé, par des éclaircies assez rapprochées, de maintenir la densité $\Sigma_{\sigma}$ au voisinage d'une valeur quelconque pendant une longue période.

Nous considérerons donc le cas d'un traitement sylvicole tel que $\Sigma_{\sigma}$ reste constant, de telle sorte que, dans (eqn 6), $\alpha$ $\left(\Sigma_{\sigma}\right)=\alpha^{\prime}$ est une constante ( $n b$ : quand la densité s'abaisse, $\alpha$ augmente). De plus, nous posons la relation : $\sigma=\varepsilon \Gamma$. Alors, on peut faire le changement de variables suivant :

$$
u=\Gamma \quad \text { et } \quad y=\frac{c}{\Gamma}
$$

qui revient à passer du temps universel $t$ à un temps biologique de la croissance $(\Gamma)$ et de la circonférence $c$ à un équivalent de vitesse moyenne, $c / \Gamma$.

On peut montrer qu'alors :

$$
\frac{d y}{d u}=\frac{-\varepsilon \alpha^{\prime}+\alpha^{\prime} y-y^{2}}{u y} \text { si } y \geq \varepsilon,-\frac{y}{u} \text { sinon. }
$$

II s'agit là d'une équation à variables séparables dont l'intégration fait appel aux fonctions hyperboliques et ne fournit qu'une solution implicite en $y$. Nous n'exposerons ici que les propriétés générales de comportement de cette solution. Le comportement dynamique de $y$ peut avoir deux types de régime, suivant le signe de la quantité :

$\Delta=\alpha^{\prime 2}-4 \varepsilon \alpha^{\prime}$ (on pose, si $\Delta \geq 0, \gamma=\frac{\sqrt{ } \Delta}{2}$ )

\section{Régime 1}

Si $\Delta<0, \mathrm{~d} y / \mathrm{du}$ est négatif et donc $y$ décroît quelle que soit la condition initiale; à partir de $y=\varepsilon$, on a $c=$ constante (fig 5 , graphique de droite).

Le comportement asymptotique théorique est donc caractérisé dans ce cas par le fait que, quelle que soit la condition initiale, la trajectoire en circonférence converge vers un point fixe; cette convergence est plus rapide si la condition initiale $c_{0}$ est proche du seuil $\varepsilon u_{0}$.

II faut préciser que cette convergence est théorique, puisque la variable $u$ ne peut pas prendre de valeurs infiniment grandes : rappelons en effet que $u=\Gamma(t)$ (cette quantité, qui a été définie comme la production totale maximale, est supposée bornée supérieurement). 

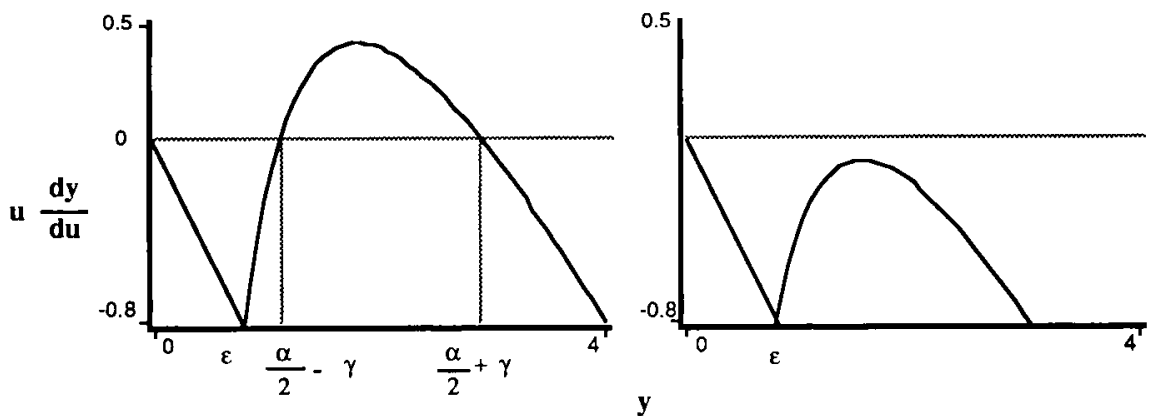

Fig 5. Comportement de $u \mathrm{~d} y / \mathrm{d} u$ en fonction de $y$ (définitions dans le texte). A gauche : $\alpha^{\prime}=4, \varepsilon=0,8$; à droite : $\alpha^{\prime}=3, \varepsilon=0,8$.

\section{Régime 2}

Si l'on suppose maintenant que $\Delta>0$, alors il existe deux points fixes pour $y$, en :

$$
y_{-}=\frac{\alpha^{\prime}}{2}-\gamma \quad \text { et } \quad y_{+}=\frac{\alpha^{\prime}}{2}+\gamma
$$

Le premier est instable et le second stable (fig 5, graphique de gauche).

Le comportement asymptotique dépend de la condition initiale $c_{0}$ et l'on peut distinguer trois régions :

$$
\text { si } c_{0}<y_{-}, \quad \text { à partir d'un instant fini } t_{\mathrm{f}} \text {, }
$$$$
\text { on a } \quad \frac{\mathrm{d} c}{\mathrm{~d} t}=0
$$

si $y_{-}<c_{0}<y_{+}, \quad y$ croît et tend vers $y_{+}$, si $y_{+}<c_{0}, \quad y$ décroît et tend vers $y_{+}$.

$\mathrm{Si}$ l'on revient maintenant au plan de phase $(\Gamma, c)$, il existe deux trajectoires singulières : $c=y_{-} \Gamma$ et $c=y_{+} \Gamma$ qui délimitent deux zones de comportement (voir fig 6) : en-dessous de $c=y_{-} \Gamma$, toutes les trajectoires finissent par être horizontales ( $c=$ constante); au-dessus, toutes les trajectoires tendent vers la droite $c=y_{+} \Gamma$ (dans ce cas, la croissance n'est limitée que par $\Gamma$ ).
Les situations conduisant au régime 1 ont un impact pratique limité et temporaire : avec le paramétrage indiqué dans le chapitre Comptabilité entre les deux niveaux, ces situations correspondent à des peuplements jeunes très denses; même si les éclaircies sont faibles, la densité, telle qu'elle est mesurée dans ce modèle, s'abaisse très vite et l'on retrouve alors un comportement conforme au régime 2. Pour le régime 2 , il existe deux régions de croissance au sein du peuplement, en fonction de la position initiale de l'arbre considéré : il se produit une séparation du peuplement en deux sous-populations distinctes par leur comportement asymptotique. Pour les arbres suffisamment dominés à l'origine, l'arrêt de la croissance est précoce (lié à leur position sociale défavorable), alors que le peuplement dominant poursuit une croissance soutenue, qui n'est progressivement ralentie que par le vieillissement général. Insistons bien sur le fait qu'il y a plus, dans cet énoncé, que ce qui pouvait être déduit simplement de la forme brute de l'équation différentielle : en effet, non seulement les arbres situés en-dessous du seuil "ne poussent plus", mais il existe en outre des situations (car $y_{-}$est toujours supérieur à $\varepsilon$ ) où la croissance est initialement non nulle mais finit par culminer (fig 6). 
II s'agit donc là d'un trait du comportement global du système, qu'on peut interpréter comme une structuration continue du peuplement en cours de croissance : en revenant à l'interprétation du chapitre Interprétation et hypothèses, on dira qu'un individu suffisamment dominé n'a pas la vigueur suffisante pour continuer à se ménager un accès à la lumière et finit donc par être «laché dans la course pour la survie». Cette interprétation rejoint la distinction classique entre peuplements principal et subordonné, en lui ajoutant un caractère dynamique.

En ce qui concerne le comportement numérique du modèle, pour des simulations à longue échéance, le fait que la vitesse de croissance en circonférence des arbres de l'étage dominant tende asymptotiquement vers la quantité $y_{+} \mathrm{d} \Gamma / \mathrm{d} t$ garantit également qu'on est à l'abri de comportements explosifs : toutes les trajectoires individuelles sont bien guidées par la fonction de l'âge $\Gamma$, qui définit la productivité du site.

Sur un plan plus méthodologique, enfin, il est certain que la mise en évidence de cette propriété qualitative du modèle n'a été rendue possible que parce que son expression est relativement simple, permettant le calcul explicite. II en serait allé tout autrement avec des formulations complexes du type des modèles de régression à grand nombre de variables (Wykoff et al, 1982), dont le comportement n'est appréhendable qu'au prix d'un nombre élevé de

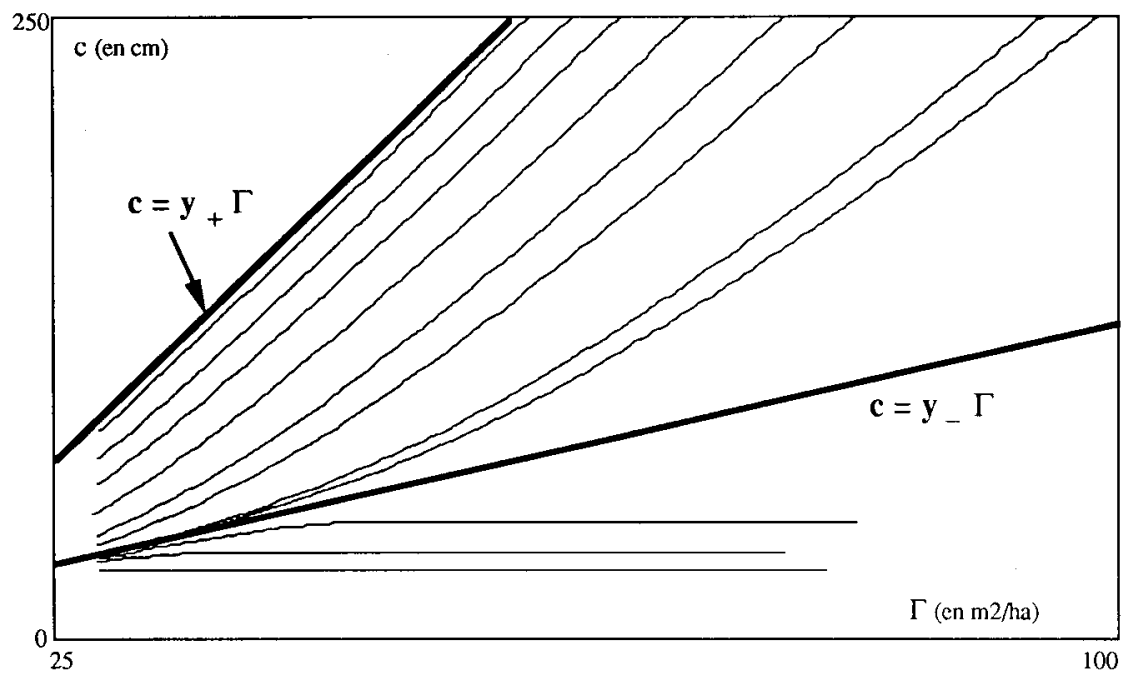

Fig 6. Simulation de trajectoires individuelles (réalisée grâce au logiciel DYNAMAC (Rousseau, 1988)). Les équations différentielles sont :

$$
\frac{\mathrm{d} \Gamma}{\mathrm{d} t}=r(K-\Gamma) \quad \text { et } \quad \frac{\mathrm{d} c}{\mathrm{~d} t}=\frac{\mathrm{d} \Gamma}{\mathrm{d} t} \alpha^{\prime}\left(1-\varepsilon \frac{\Gamma}{c}\right) \quad \text { si } c \geq \varepsilon \Gamma, \quad 0 \text { sinon }
$$

avec les paramètres : $K=134 \mathrm{~m}^{2} \mathrm{ha}^{-1}, r=110^{-2} \mathrm{an}^{-1}, \alpha^{\prime}=6 \mathrm{~cm} \mathrm{~m}^{-2}$ ha, $\varepsilon=0.8 \mathrm{~cm} \mathrm{~m}^{-2}$ ha. Sont figurées, dans le plan de phase $(\Gamma, c)$, quelques trajectoires, à partir de diverses conditions initiales; on a repéré les deux trajectoires singulières : $c=y_{-} \Gamma$ (instable) et $c=y_{+} \Gamma$ (stable). 
simulations. Cette étude apporte donc un argument supplémentaire pour insister sur le caractère éminemment souhaitable de modèles mathématiquement simples, même et (devrait-on dire) surtout lorsqu'on modélise des systèmes complexes et mal connus dans le détail de leur fonctionnement.

\section{VALIDATION DU MODĖLE SUR DES DONNÉES EXTÉRIEURES}

Les données prises en compte pour la construction et l'identification du modèle étaient relativement étroites : en particulier, les traitements ne présentaient qu'un intervalle réduit de densité, il n'y avait que peu de données individuelles dans le jeune âge (entre 30 et 60 ans) et, enfin, les dispositifs étaient tous situés sur des stations a priori favorables. Comme on l'a souligné en introduction, ce plan expérimental n'est pas particulièrement adapté aux perspectives d'utilisation du modèle : un premier jeu de tests a donc été effectué, afin d'apprécier la validité du modèle (Dhôte, 1990, 155-180). Nous présenterons ici deux études, la première destinée à tester la qualité prédictive du modèle pour un ensemble de peuplements présentant un large gradient de densités, la seconde ayant révélé des particularités intéressantes du comportement au voisinage d'une sévère crise de croissance liée à un déficit hydrique.

La procédure est la suivante : on dispose d'une série d'observations relevées dans un groupe de placettes traitées différemment, sur une longue période (40 à 50 ans, ici); on se donne la situation initiale effectivement observée, à partir de laquelle on simule la croissance sur toute la période d'observation, en spécifiant des éclaircies aussi proches que possible de celles pratiquées (nature, intensité et rota- tion), enfin on compare les résultats de simulation aux valeurs observées.

Dans les deux applications qui seront présentées, on ne connaît que les grandeurs au niveau du peuplement (nombre de tiges, surface terrière, circonférence dominante, etc) et éventuellement la série des inventaires par classes de circonférence; il ne sera donc pas possible d'évaluer l'erreur sur les trajectoires individuelles, mais on peut donner une bonne image synthétique de la qualité des prévisions à l'aide de la circonférence dominante, en supposant que cette statistique résume bien, à un moment donné, la population des arbres qui seront finalement récoltés. Pour "rentrer" dans le modèle, on générera une liste d'arbres fictifs compatible avec l'inventaire initial par classes de circonférence.

En ce qui concerne le paramétrage du modèle, on doit spécifier la valeur des paramètres locaux intervenant dans la fonction $\Gamma$ de production totale maximale (ie "localiser" le modèle) : comme il existe toujours au moins un traitement à forte densité, on peut s'attendre à ce que sa production soit très peu différente de $\Gamma$, si le modèle est valide.

\section{Qualité de la réponse prédite à la densité}

En ce qui concerne l'évaluation de la qualité prédictive du modèle, notamment pour la réponse à la densité aux deux niveaux de l'arbre et du peuplement, l'expérience danoise de Totterup (Bryndum, 1987) est tout à fait indiquée : elle est basée, en effet, sur un gradient de densités très large (8 traitements), maintenu pendant 40 ans par des éclaircies très rapprochées $(2$ ans entre 28 et 44 ans, 4 ans de 44 à 56 ans et 6 ans jusqu'à 68 ans). En moyenne sur la période $19-68$ ans, les surfaces terrières 
sur pied et les accroissements annuels en surface terrière s'établissent aux valeurs mentionnées dans le tableau II.

La valeur indiquée pour le traitement $A$ étant vraisemblablement un accroissement net (hors mortalité), on constate que la croissance est sensiblement identique pour les modalités $A$ à $C$, c'est-à-dire pour des surfaces terrières comprises entre 50 et $100 \%$ d'un témoin non éclairci, alors qu'elle chute de façon appréciable en dessous ( $D$ à $F$ ). Dans le même temps, la croissance individuelle s'accélère de façon spectaculaire avec l'intensité des éclaircies : à 68 ans, le diamètre dominant est de $32,6 \mathrm{~cm}$ pour $A$, mais $63,3 \mathrm{~cm}$ pour $F$ (Bryndum, 1987).

Nous avons simulé, sur la période 2868 ans, les trois traitements $B, D$ et $F$, en prenant pour production totale maximale $\Gamma$, caractérisant le site, une fonction ajustée sur les valeurs observées pour $B$, et, comme situation initiale, une distribution de circonférences sensiblement compatible avec les statistiques de peuplement disponibles. Les résultats sont résumés dans le tableau III.

La figure 7 représente l'évolution au cours du temps des 2 variables les plus si- gnificatives : production totale en surface terrière et circonférence dominante, ce qui permet d'illustrer comment les erreurs finalement constatées sont acquises. D'un point de vue quantitatif, les prédictions sont trop "optimistes", en ce qui concerne la production totale : en accroissement annuel sur la période, on prédit des pertes qui, par rapport à $B$, s'élèvent respectivement à 11 et $34 \%$ pour $D$ et $F$, contre 19 et $53 \%$ observés. Pour les circonférences dominantes (pour lesquelles nous ne disposons malheureusement que de 3 valeurs observées), la prédiction est correcte pour le traitement intermédiaire (D), alors que l'accroissement est sous-estimé pour le peuplement dense et surestimé pour le peuplement très clair.

En première approche, on pourrait attribuer cette double structure des erreurs au paramétrage : en admettant que le modèle est structurellement adéquat, un mauvais paramétrage de la réponse à la densité $\alpha$ serait responsable à la fois d'une surestimation de l'accroissement du peuplement et d'un biais sur l'accroissement individuel. Ces considérations apportent donc un éclairage supplémentaire à l'articulation, interne au modèle, des deux niveaux : les contraintes qui déterminent la structure du

Tableau II. Surface terrière sur pied et accroissement annuel en surface terrière, pour les 8 traitements du dispositif expérimental de Totterup (Bryndum, 1987). Les valeurs indiquées sont des moyennes sur la période 19-68 ans.

\begin{tabular}{lcccccccc}
\hline Traitement & $A$ & $B$ & $B-C$ & $C$ & $D$ & $D-E$ & $E$ & $F$ \\
\hline $\begin{array}{l}\text { G moyenne } \\
\left.\text { (en } \mathrm{m}^{2} \mathrm{ha}^{-1}\right)\end{array}$ & 35,41 & 28,55 & 24,53 & 19,13 & 14,99 & 12,66 & 10,81 & 8,70 \\
$\Delta G$ & 1,17 & 1,24 & 1,23 & 1,25 & 1,15 & 1,07 & 0,94 & 0,79 \\
$\frac{\Delta}{\Delta t}$ moyen & & & & & & & & \\
$\left(\mathrm{en} \mathrm{m}^{2} \mathrm{ha}^{-1} \mathrm{an}^{-1}\right)$ & & & & & & & & \\
\hline
\end{tabular}


Tableau III. Simulation de trois traitements du dispositif de Totterup (Bryndum, 1987). Valeurs à 68 ans : nombre de tiges $N$, surface terrière $G$, circonférence dominante $c 0$ et production totale $G T$; accroissement annuel moyen en surface terrière entre 28 et 68 ans : $\Delta G / \Delta t$. Les valeurs dites "observées" sont réévaluées à partir des graphiques de Bryndum.

\begin{tabular}{|c|c|c|c|c|c|c|}
\hline \multirow[t]{2}{*}{ Variable } & \multicolumn{2}{|c|}{ Traitement $B$} & \multicolumn{2}{|c|}{ Traitement $D$} & \multicolumn{2}{|c|}{ Traitement $F$} \\
\hline & Observée & Prédite & Observée & Prédite & Observée & Prédite \\
\hline$N($ en ha-1) & 675 & 681 & 98 & 98 & 31 & 28 \\
\hline$G\left(e n m^{2} h^{-1}\right)$ & 38,37 & 38,11 & 17,95 & 18,26 & 9,76 & 10,90 \\
\hline$C O(e n c m)$ & 111,2 & 99,4 & 151,7 & 153,0 & 198,9 & 221,2 \\
\hline$G T\left(e n m^{2} \mathrm{ha}^{-1}\right)$ & 71,52 & 71,50 & 63,50 & 66,77 & 48,67 & 56,04 \\
\hline & & & & & & \\
\hline$-\left(e n m^{2} h a^{-1} a^{-1}\right)$ & 1,086 & 1,122 & 0,885 & 1,004 & 0,515 & 0,735 \\
\hline
\end{tabular}

modèle (les traits de comportement qu'on a cherché à traduire et la forme mathématique adoptée pour assurer la cohérence entre les deux niveaux) conduisent, si le modèle est mal ajusté, à des erreurs en quelque sorte duales l'une de l'autre.

Toutefois, les principales divergences entre traitements sont bien restituées qualitativement : aux densités très faibles, on prédit, conformément à ce qui est observé, une forte stimulation de l'accroissement en circonférence des arbres résiduels et une perte de production globale, à l'échelle du peuplement.

Pour conclure, on peut admettre que ces résultats sont relativement satisfaisants, si l'on considère la faible amplitude des traitements pris en compte pour la construction et l'ajustement du modèle : rappelons que la totalité des placettes utilisées se trouvait dans un intervalle équivalent aux traitements $A-C$ de ce dispositif, et la simulation des modalités $D$ et $F$ constitue donc une très forte extrapolation; c'est dans ce contexte qu'il taut replacer le comportement qualitativement satisfaisant et l'amplitude des erreurs constatées ici.

\section{Limites de l'interprétation par la compétition aérienne}

L'étude que nous allons présenter maintenant porte sur un dispositif installé en 1923 en forêt de Darney, lieu dit Sainte-Marie (Vosges), dont le protocole et les résultats ont été présentés par Arbonnier en 1958. Ces trois placettes de 1 ha ont fait l'objet des traitements suivants : à l'exception de la rotation ( 3 et 6 ans, respectivement), les placettes 1 et 2 ont en fait connu des évolutions très similaires : prélèvements intenses jusqu'en 1929, à la fois par le haut et par le bas, puis éclaircies plus faibles (capitalisation); la placette 3 semble être restée un peu plus dense, ce qui est dû au fait que le sous-étage $y$ a été préservé plus longtemps (fig 8). Dans l'étage dominant, la croissance est très vive et la circonférence dominante suit une évolution très proche dans les 3 cas $(110 \mathrm{~cm}$ environ à 78 ans, en 1966).

L'intérêt de ce dispositif, du point de vue de la validation du modèle, ne réside pas tant dans l'amplitude des traitements, qui ont produit des peuplements très voisins, 

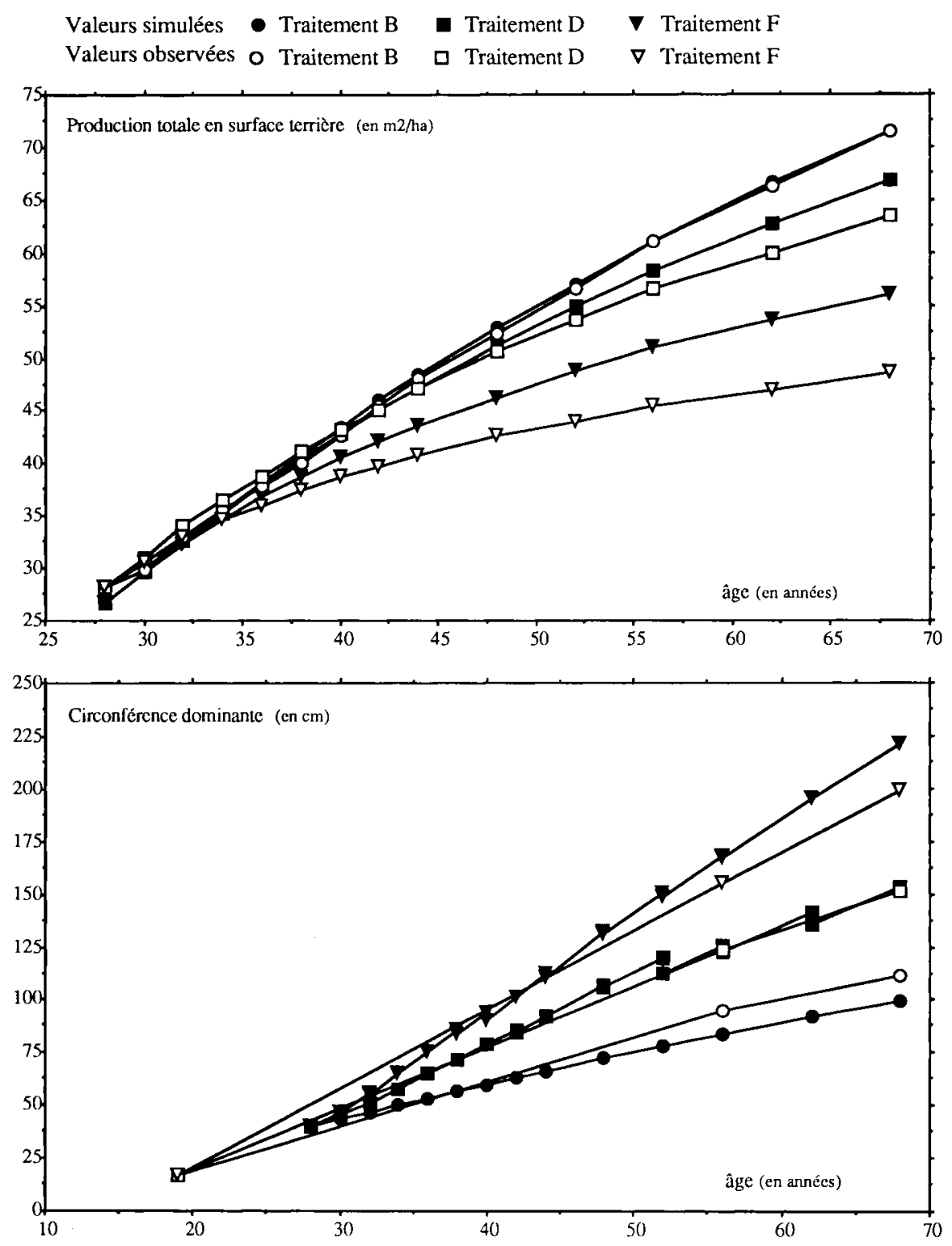

Fig 7. Simulation de 3 traitements testés dans le dispositif expérimental de Totterup (Bryndum, 1987) : B (éclaircies faibles), D (éclaircies très fortes), $F$ (éclaircies extrêmement fortes). De haut en bas, sont figurées la production totale en surface terrière et la circonférence dominante. 


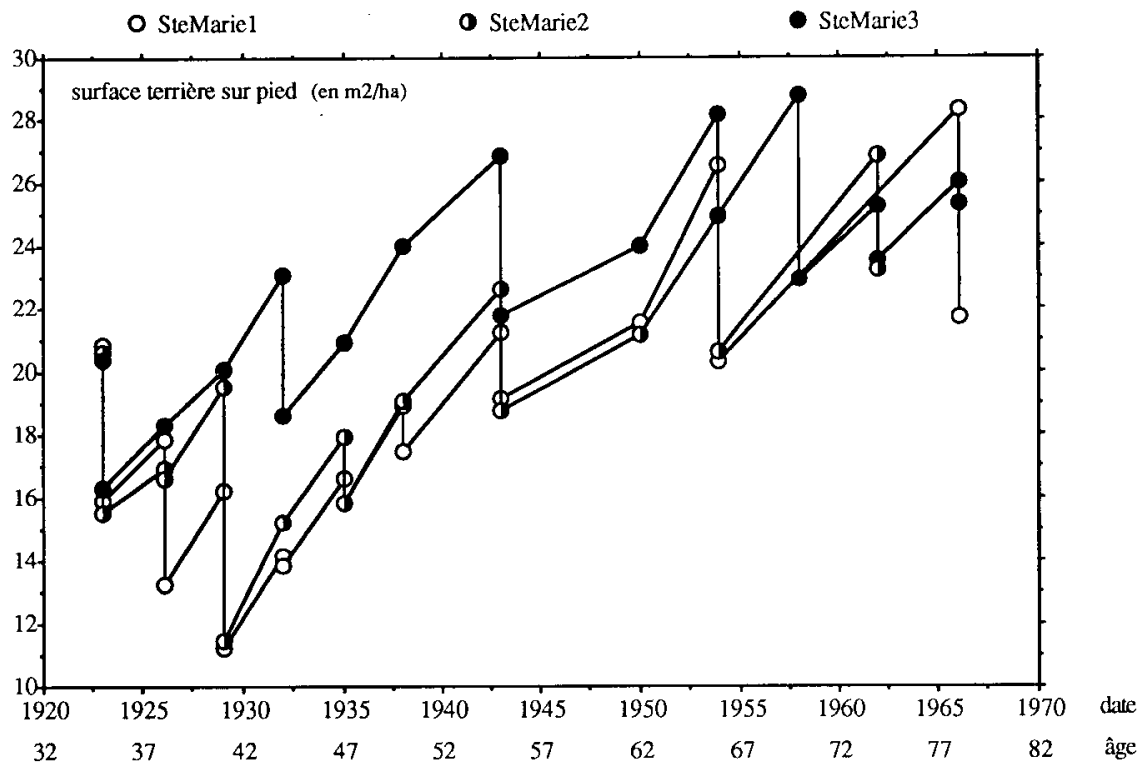

Fig 8. Évolution des surfaces terrières sur pied, pour les 3 traitements du dispositif expérimental de Sainte-Marie (Darney, Vosges).

que dans un trait inattendu au départ, à savoir la crise de croissance très prononcée traversée entre 1943 et 1950 : en effet, la vitesse de croissance chute de moitié entre 1937 et 1950 , pour se redresser spectaculairement ensuite (fig 8). C'est grâce à ce profil caractéristique (croissance vive, puis crise, puis reprise), et aux modifications concomitantes de la croissance individuelle en fonction du statut social, que nous avons abouti à des résultats très intéressants en ce qui concerne la validité du modèle.

Afin de répercuter dans la simulation la crise de croissance 1943-1950, nous avons utilisé comme fonction $\Gamma$, dans le modèle, les valeurs observées de la production totale, pour chacune des 3 placettes. La figure 9 illustre les résultats ob- tenus en ce qui concerne la croissance de la circonférence dominante; on retrouve bien la chute momentanée de la croissance, mais ce qui ressort le plus nettement de ce graphique est la structure temporelle des erreurs de prédiction; la vitesse de croissance des dominants est surestimée systématiquement lorsque la croissance générale (ie du peuplement) est rapide, à la fois entre 1926 et 1937 et à la reprise de 1950-1954; au contraire, la prédiction est correcte quand la vitesse est faible (crise de 1943-1950).

Pour mieux cerner le phénomène, nous avons fait une expérience indépendante qui visait à estimer les valeurs du seuil $\sigma$ de croissance minimale, sur les données brutes des 3 placettes. En l'absence de données individuelles (les arbres n'ont pas 
été numérotés), nous avons, pour cela, comparé les fonctions de répartition en circonférence pour deux dates successives; si le modèle structurel (eqn 1) est valide, ces fonctions de répartition doivent être identiques en-dessous de $\sigma$, puis diverger au-delà. Cette expérience s'est avérée concluante et a fourni des séries d'estimations graphiques de $\sigma$ que nous appellerons "valeurs observées".

La figure 9 montre que, conformément aux lois de comportement imposées au modèle, les valeurs simulées de $\sigma$ augmentent régulièrement avec l'âge. Par contraste, les valeurs observées de $\sigma$ présentent une structure temporelle très différente, et qui prend tout son sens quand on la met en regard avec la vitesse de croissance générale du peuplement : chaque fois que la croissance est rapide, $\sigma$ descend vers des valeurs particulièrement basses (5 à $15 \mathrm{~cm}$ en 1929, 1935 et 1950 !), autrement dit la croissance est significative pour pratiquement toutes les classes sociales; au contraire, en période de crise, la croissance cesse brutalement pour les arbres dominés et $\sigma$ "remonte" à des valeurs plus fortes, conformes aux prédictions du modèle.

En somme, le profil des erreurs de prédiction sur $\sigma$ est identique à celui sur l'accroissement de la circonférence dominante. On peut le comprendre en analysant la sensibilité du modèle à $\sigma$; sous les contraintes fixées, on prédit correctement l'accroissement au niveau du peuplement $\mathrm{d} G / \mathrm{d} t$; si le scénario observé est correctement simulé (en nombre de tiges $N$ et surface terrière $G$ ), alors le point moyen du nuage des accroissements individuels $(\bar{c}, 1 / N \mathrm{~d} G / \mathrm{d} t)$ est toujours correctement prédit ( $\bar{c}$ étant la circonférence moyenne du peuplement); il s'ensuit que l'erreur moyenne pour une valeur de circonférence donnée est de type "pivotant»: si l'on surestime $\sigma$, on sures- time également la vitesse de croissance des individus tels que $c>\bar{c}$ et l'on sousestime celle des individus tels que $c<\bar{c}$; l'erreur est d'autant plus grande qu'on se trouve plus loin de la circonférence moyenne. L'erreur de prédiction sur les dominants apparaît bien, dans ces conditions, comme imputable à une mauvaise prédiction du seuil $\sigma$.

Cette expérience apporte beaucoup plus d'informations sur la validité du modèle que la précédente, dans la mesure où ce n'est plus uniquement le paramétrage qui est remis en cause, mais bien les lois de comportement internes et avec elles les hypothèses sous-jacentes : au vu d'un premier jeu de données, on avait imposé à $\sigma$ de croître régulièrement avec l'âge et interprété ce phénomène en disant que $\sigma$ représente la taille minimum d'un arbre du couvert principal, ayant accès à la lumière, taille qui doit augmenter avec le développement général du peuplement.

Une hypothèse aussi sommaire ne suffit plus à expliquer les fortes variations de $\sigma$ constatées ici, d'autant que celles-ci coïncident avec celles de la croissance générale du peuplement; on pourrait dire que, localement, au voisinage d'une dépression ou d'une reprise, toutes les classes d'arbres n'accusent pas dans une même proportion les à-coups : pendant la crise, les dominants «marquent le pas», mais les dominés réagissent proportionnellement plus fort, en cessant toute croissance; à la reprise, c'est l'ensemble des classes sociales qui bénéficient de la «croissance retrouvée".

Cette forme de relation statut socialvitesse de croissance suggère fortement l'intervention du facteur alimentation hydrique, en liaison avec un modèle d'occupation de l'espace souterrain. Si les systèmes racinaires sont stratifiés de façon symétrique à l'appareil foliaire (Delvaux, 1966; Abetz, 1988), c'est-à-dire avec des 


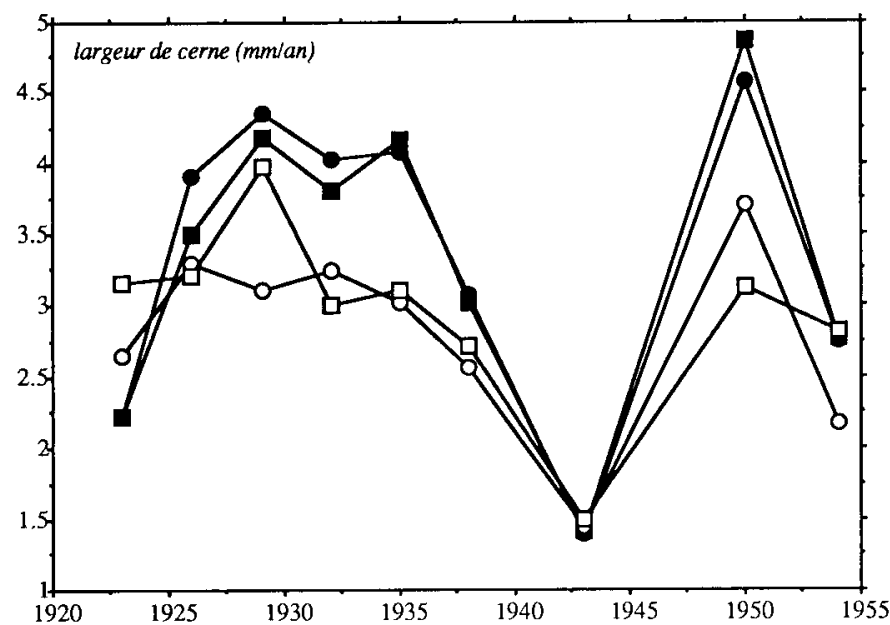

Valeurs simulées:

- SteMarie 1

- SteMarie 2

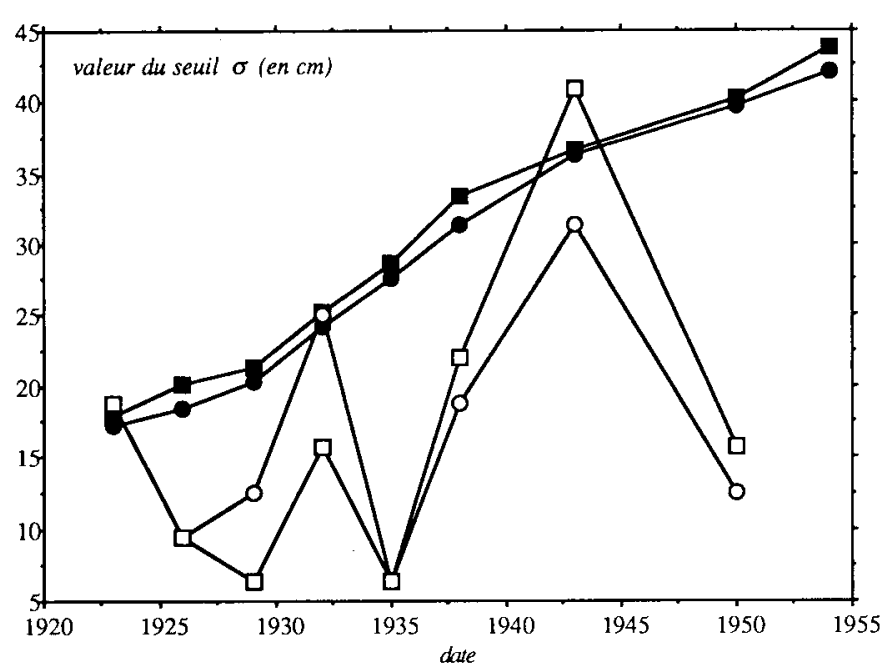

Valcurs observées :

o SteMarie 1

口 SteMarie 2

Fig 9. Valeurs observées et simulées pour deux placettes du dispositif expérimental de Sainte-Marie (Darney, Vosges). En haut, accroissement annuel (moyennes périodiques) de la circonférence dominante (transformé en largeur de cerne); en bas, valeurs de la circonférence-seuil $\sigma$ en dessous de laquelle la croissance individuelle est nulle.

arbres dominants explorant une plus grande profondeur de sol, au détriment des dominés cantonnés aux horizons superficiels, un stress hydrique prononcé risque d'avoir un effet très dissymétrique, selon le statut social : arrêt complet de la croissance de tous les individus dominés, dont l'alimentation en eau est trop faible, alors que les dominants trouvent encore dans les horizons profonds assez d'eau pour préserver une croissance significative. 
L'intervention des facteurs hydriques est étayée par deux constats indépendants : la crise de croissance de la décennie 1940, en Lorraine, apparaît très nettement dans les placettes permanentes de production (Haye, Darney) ainsi que dans les études de dendrochronologie menées actuellement par le laboratoire de Phytoécologie de I'INRA Champenoux (JF Picard et JL Dupouey, communication personnelle); les données météorologiques du poste de Nancy indiquent une période exceptionnellement prolongée de déficit des pluies en saison de végétation. II est possible qu'on assiste, en outre, dans cette expérience, à un effet conjoint sylviculture-milieu : les peuplements étant très ouverts après les éclaircies intenses de 1923 à 1929, le facteur lumière n'est plus limitant, temporairement, ce qui amplifierait l'effet des facteurs hydriques.

Bien que le tour de la question ne soit pas terminé, on peut d'ores et déjà souligner l'importance, aussi bien pratique que théorique, de ce résultat : du point de vue sylvicole, il est évidemment utile de connaître les différences de réaction entre milieux à un traitement donné, afin d'adapter les modes de conduite aux conditions écologiques (structure et profondeur de sol, etc); en ce qui concerne la compréhension du fonctionnement, il paraît souhaitable maintenant, au moins comme étape intermédiaire dans la construction de modèles assez globaux comme celui que nous proposons ici, de se rapprocher de formulations plus fonctionnelles qui prennent en compte divers phénomènes : photosynthèse nette, économie de l'eau en fonction du stade de développement, partition de la biomasse entre organes, stratégies spécifiques de développement souterrain (Cannel, 1985; McMurtrie, 1985).

\section{DISCUSSION ET CONCLUSION}

À travers les résultats qui viennent d'être exposés, trois types d'enseignement d'ordre méthodologique ressortent : la méthode générale de modélisation, avec notamment une phase importante qui est la formulation d'hypothèses explicites; l'intérêt de formulations mathématiques simples, qui permettent une étude aussi rigoureuse que possible du comportement du modèle; une méthode de traitement de l'articulation entre deux niveaux de fonctionnement, en l'occurrence l'arbre et le peuplement. Ces trois points correspondent à des choix délibérés de méthodes, clairement motivés dès le départ par le double souci d'arriver à une évaluation correcte des performances, du comportement et de la généralité des modèles, et surtout d'une bonne insertion de ces modèles dans un processus continu d'expérimentation destiné à améliorer leur validité et leur fiabilité.

\section{Méthode générale}

À partir d'une analyse des données de croissance individuelle, on a dégagé des structures régulières concernant : a) l'effet de la densité sur la croissance du peuplement, b) l'effet du rang social, de l'âge et de la densité du peuplement sur la croissance individuelle. Ces structures empiriques suggèrent une interprétation basée sur le fonctionnement des interactions compétitives entre classes sociales pour l'accès à une même ressource, la lumière. En sens contraire, cette interprétation conduit à postuler des règles de comportement plus générales que la structure empirique de départ : on passe ainsi d'un mo- 
dèle descriptif à un modèle plus biologique, ou encore plus "théorique". Le recours à des hypothèses améliore la généralité du modèle et permet de surmonter partiellement les insuffisances de la base expérimentale. La validité de ce schéma a été testée favorablement, d'un point de vue qualitatif, sur des données extérieures (Qualité de la réponse prédite à la densité).

Cependant, indépendamment de l'interprétation donnée, la structure reste avant tout un trait géométrique simple, et à ce titre susceptible d'expérimentation : c'est ainsi que la notion de seuil introduite au chapitre Croissance individuelle et rang social : effet de seuil a pu être testée au chapitre Limites de l'interprétation par la compétition aérienne en parallèle aux simulations du modèle. II est certain que si I'on avait adopté, comme modèle d'arbre, une formulation continue non linéaire (Alder, 1979) au lieu d'un modèle linéaire segmenté autour d'une valeur seuil, il aurait été beaucoup plus difficile de comprendre les erreurs de prédiction et surtout de les interpréter.

\section{Simplicité mathématique}

Le modèle d'arbre consiste en une équation différentielle du premier ordre où interviennent, composés additivement et multiplicativement, les effets de l'âge, de la densité, de la dimension et du statut social. La structure linéaire de l'équation facilite le traitement des deux niveaux : on peut former par sommation, à partir du modèle d'arbre, un modèle de peuplement équivalent, faire apparaître des quantités dont l'interprétation est simple et conditionner les formulations aux deux niveaux de telle sorte qu'elles respectent des contraintes fixées a priori.

De plus, la simplicité des équations est une condition impérative si l'on veut étu- dier explicitement les propriétés asymptotiques des solutions : on a montré que les trajectoires individuelles se classent en deux groupes, suivant les conditions initiales (Comportement mathématique : séparation du peuplement en deux souspopulations); ce comportement de structuration continue du système-peuplement en deux étages (certains individus initialement dominants étant progressivement relégués dans le sous-étage) est cohérent avec les connaissances actuelles sur la dynamique des structures sociales, dans les peuplements équiennes, et constitue une première validation qualitative du modèle.

\section{Articulation entre deux niveaux d'organisation}

On a cherché à modéliser simultanément, et de façon cohérente, les deux niveaux de l'arbre et du peuplement : la densité influe globalement sur la production du peuplement, selon une loi de saturation, et localement sur chaque individu de l'étage dominant. Ce traitement conjoint instaure un lien très fort entre les paramétrages aux deux niveaux et conduit à limiter la souplesse du modèle.

Le passage arbre-peuplement a également suggéré l'introduction d'une mesure de densité peu courante, mais dont la définition est intéressante du point de vue des phénomènes de compétition sous-jacents : cette quantité (Compatibilité entre les deux niveaux) est une statistique calculée pour les seuls arbres du peuplement dont la dimension est supérieure à un certain seuil. Ainsi est renforcée la nature «à deux compartiments" du système modélisé : l'existence d'une dimension seuil subdivise le peuplement en deux strates, ou horizons de compétition, étage dominant et étage subordonné; la compétition intervient entre individus de l'étage dominant, d'une part, 
et d'autre part par oppression unilatérale de l'étage subordonné par l'étage dominant (one-sided competition, Ford et Diggle, 1981).

En ce qui concerne la nature des phénomènes qui déterminent la croissance individuelle et le développement de la structure des peuplements, les fortes structures mises en évidence confirment l'importance des facteurs sociaux. La dimension d'un individu, dans un peuplement donné, est un intégrateur de toute son histoire compétititive et, de fait, est très souvent reconnue comme le meilleur prédicteur de son accroissement futur, avant tout indice de compétition «horizontale» (Perry, 1985). On a constaté également que l'amélioration du potentiel de croissance individuel n'est pas régulière sur tout l'intervalle des statuts sociaux, mais passe par une articulation brutale, de type seuil, au voisinage de la base de l'étage principal de végétation.

À partir de constats analogues, sur divers types de monocultures annuelles ou pérennes, d'autres auteurs (Ford, 1975, Ford et Diggle, 1981) ont insisté sur le rôle fondamental du processus de compétition pour la lumière dans la constitution et l'accentuation des hiérarchies sociales. Les résultats présentés ici corroborent assez largement ces conclusions; toutefois, le comportement très suggestif des peuplements analysés au chapitre Limites de l'interprétation par la compétition aérienne indique que, soit en période de crise profonde, soit plus durablement sur station difficile, il est très probable que la compétition pour d'autres ressources (eau, minéraux) devient prépondérante : c'est ce que tendraient à montrer des études récentes menées à l'occasion du dépérissement des forêts (Abetz, 1988). Dans la perspective actuelle de sylviculture d'arbres, l'identification des facteurs de production limitants est essentielle, aussi bien pour guider le choix de traitements adaptés au milieu que pour améliorer la capacité prédictive des modèles de croissance. Dans ce contexte, des modèles tels que celui que nous avons présenté, comme référence "hypothèse nulle», peuvent permettre de poser les questions, mais un traitement approprié demandera certainement un effort important d'expérimentation et d'élaboration théorique.

\section{RÉFÉRENCES}

Abetz P (1988) Untersuchungen zum Wachstum von Buchen auf der Schwäbischen Alb Allg Forst Jagdztg, $159 \mathrm{Jg}, 11 / 12,215-223$

Alder J (1979) A distance dependant tree model for exotic conifer plantations in East Africa. For Sci 25, 1, 59-71

Arbonnier $P$ (1958) Les places d'expériences de Sainte-Marie en Forêt Domaniale de DarneyMartinvelle. Bull Soc For Franche-Comté 3, 3-15

Assmann E (1970) The principles of forest yield study. Pergamon Press, $503 \mathrm{p}$

Aussenac G, Granier A, Naud R (1982) influence d'une éclaircie sur la croissance et le bilan hydrique d'un jeune peuplement de Douglas (Pseudotsuga menziesii (Mirb) franco). Can J For Res 12, 2, 222-231

Bouchon J, Dhôte JF, Lanier L (1989) Réaction individuelle de hêtres (Fagus silvatica L) d'âges divers à diverses intensités d'éclaircie. Ann Sci For 46, 3, 251-259

Braathe $P$ (1957) Thinnings in even-aged stands. A summary of European literature. Fac For, Univ of $\mathrm{N}$ Brunswick, Fredericton, $92 \mathrm{p}$

Bryndum $H$ (1987) Buchendurchforstungsversuche in Dänemark. Allg Forst Jagdztg, 158 $\mathrm{Jg}, 7 / 8,1.15-121$

Cannell MGR (1985) Dry matter partitioning in tree crops. In: Attributes of trees as crop plants (Cannell, Jackson, eds) Inst Terrestrial Ecology, Penicuik, Scotland, 160-193

Curtis RO, Marshall DD (1986) Levels-ofgrowing-stock cooperative study in Douglas fir Rep $n^{\circ}$ 8-The LOGS study: twenty-year 
results. USDA For Serv, Res Pap, Pac N-W Res Station, $113 p$

Delvaux J (1966) Contribution à l'étude de l'éducation des peuplements. IV. La compétition au niveau des classes sociales. St de Rech des $E$ et $F$, Groenendaal-Hoeilaart, TravauxSérie $B, 32,48 p$

Dhôte JF (1990) Modèles de la dynamique des peuplements forestiers : articulation entre les niveaux de l'arbre et du peuplement. Applications à la sylviculture des hêtraies. Thèse, Laboratoire de Biométrie, Univ C BernardLyon I, Lyon, $256 \mathrm{p}$

Ferrand JC (1982) Étude des contraintes de croissance. 2. Variabilité en forêt des contraintes de croissance du hêtre. Ann Sci For 39, 3, 187-217

Ford ED (1975) Competition and stand structure in some even-aged monocultures. $J$ Ecol 63 , 311-333

Ford ED, Newbould PJ (1970) Stand structure and dry weight production through the sweet chesnut (Castanea sativa Mill) coppice cycle. $J$ Ecol 58, 275-296

Ford ED, Diggle PJ (1981) Competition for light in a plant monoculture modelled as a spatial stochastic process. Ann Bot (Lond) 48, 481 500

Houllier F (1986) Échantillonnage et modélisation de la dynamique des peuplements forestiers; application au cas de l'Inventaire Forestier National. Thèse, Laboratoire de Biométrie, Univ C Bernard-Lyon I, Lyon, 267 p

Houllier F, Bouchon J, Birot $Y$ (1991) Modélisation de la dynamique des peuplements forestiers : état et perspectives. Rev For Fr XLIII, 2, 87-108

Johnston DR, Grayson AJ, Bradley RT (1967) Forest planning. Faber \& Faber Ltd, London, $541 \mathrm{p}$

Keller R, Timbal J, Le Tacon F (1976) La densité du bois de hêtre dans le Nord-Est de la France. Influence des caractéristiques du milieu et du type de sylviculture. Ann Sci For 33, 1, 1-17

Landsberg JJ (1986) Physiological ecology of forest production. Academic Press, $198 \mathrm{p}$

Langsæter A (1941) Om tynning i enaldret granog furuskog. Medd Nor Skogsforsøgsves, VIII, $131-216$
Long JN (1985) A practical approach to density management. For Chron, 23-27

McMurtrie RE (1985) Forest productivity in relation to carbon partitioning and nutrient cycling: a mathematical model. In: Attributes of trees as crop plants (Cannell, Jackson, eds) Inst Terrestrial Ecology, Penicuik, Scotland, 194-207

Maugé JP (1975) Modèles de croissance et de production des peuplements modernes de Pin maritime. AFOCEL, Nangis, 227-250

Meller CM (1954) The influence of thinning on volume increment. In: Thinning problems and practices in Denmark. World For Ser, Bull $n^{\circ}$ 1, Techn Pub 76

Munro DD (1974) Forest growth models: a prognosis. In: Growth models for tree and stand simulation. IUFRO, Vienne, Inst för Skogsproduktion, Rapporter och Uppsater, Res Notes, 30, 7-20

Oswald H (1971) La forêt domaniale de Retz. Doc Int INRA-CNRF, Champenoux

Oswald H (1981) Le carré latin de Souilly. Dispositif expérimental d'éclaircie du hêtre. Doc Int INRA-CNRF, Champenoux

Oswald H, Divoux A (1978) Dispositif expérimental d'éclaircie du hêtre "Camp Cusson" en forêt domaniale d'Eawy. Doc Int INRACNRF, Champenoux

Pardé J (1964) Intensité des éclaircies et production ligneuse. Rev For Fr 12, 936-945

Pardé J (1981) De 1882 à 1976/1980 : les places d'expérience de sylviculture du hêtre en forêt domaniale de Haye ( $M$ et $M$ ). Rev For Fr 33, 41-64

Perry DA (1985) The competition process in forest stands. In: Attributes of trees as crop plants (Cannel, Jackson, eds) Inst Terrestiral Ecology, Penicuik, Scotland, 481-506

Polge $H$ (1973) État actuel des recherches sur la qualité du bois de hêtre. Bull Tech Off Nat For Fr Fontainebleau, 13-22

Polge $H$ (1981) Influence des éclaircies sur les contraintes de croissance du hêtre. Ann Sci For 38, 4, 407-423

Rousseau B (1988) Vers un environnement de résolution de problèmes en biométrie. Apport des techniques de l'intelligence artificielle et 
de l'interaction graphique. Thèse, Univ $C$ Bernard-Lyon I, Lyon, $281 \mathrm{p}$

Schober R (1972) Die Rotbuche. JD Sauerländer's Verlag, $333 p$

Schütz JP (1981) L'éclaircie sélective de Schädelin; évolution et pratique actuelle. Rev For $\operatorname{Fr} 33,7-18$
Whyte AGD, Woolons RC (1990) Modelling stand growth of radiata pine thinned to varying densities. Can J For Res 20, 1069-1076

Wykoff WR, Crookston WL, Stage AR (1982)

User's guide to the stand prognosis model, USDA For Serv Gen Tech Rep, INT-133, $112 p$ 